
Changes in the treatment of breast cancer

\author{
Madrid, Spain \\ 20-22 June 2007
}

Received: 23 May 2007 Published: 19 June 2007

(C) 2007 BioMed Central Ltd

\section{Speaker Abstracts}

\section{Introduction}

\section{S1}

\section{Assessing prognosis for early breast cancer: clinical versus genetic profiles GN Hortobagyi}

Department of Breast Medical Oncology, The University of Texas MD Anderson Cancer Center, Houston, TX, USA

Breast Cancer Research 2007, 9(Suppl 1):S1 (doi: 10.1186/bcr1684)

Since the introduction of systemic adjuvant chemotherapy (ACT) and endocrine therapy in the early 1970s, the determination of risk of recurrence and death from breast cancer became a critical piece of information in the selection of the optimal postoperative treatment strategy. Classical histopathological prognostic factors included tumor size, regional lymph node metastases and number of axillary nodes involved, tumor grade, presence of lymphovascular invasion, and, more recently, estrogen receptor (ER) and progesterone receptor status, measurement of proliferative activity (S-phase fraction, mitotic index, $\mathrm{Ki}-67$ ), and HER2 overexpression/amplification. As isolated factors, they have limited predictive ability in the case of individual patients. For that reason, prognostic indices were developed. The most successful is Adjuvant!Online, an online nomogram developed by Peter Ravdin. This nomogram incorporates tumor size, axillary nodal status, tumor grade, ER status, age and comorbidity. The nomogram will provide an assessment of recurrence and mortality rates at 10 years, including deaths due to comorbid conditions. In addition, the nomogram also calculates relative and absolute benefit from various adjuvant interventions: tamoxifen, aromatase inhibitors, and first-generation, secondgeneration and third-generation ACT regimens. The prognostic and predictive value of this nomogram has been externally validated, with a margin of error $\leq 1 \%$. Over the past decade, high-throughput technologies have been developed based on gene expression profiling. These include between two and a couple of hundred genes, and have the ability to separate patients with excellent outcomes from those with higher risk. One of these prognostic profiles has been externally validated and is currently undergoing testing for clinical utility in a large, multicenter, prospective randomized trial (MINDACT). Another approach was based on prospectively identifying a set of genes from the literature and from the results of gene expression profiling. Mathematical modeling then led to the selection of 16 genes related to cell proliferation, ER-driven genes, HER2 and proteases, as well as five 'housekeeping' genes (OncotypeDx). This assay is based on RT-PCR, is reproducible and applicable to archival, paraffin-embedded material, and has been shown to predict prognosis in patients with lymph-nodenegative, ER-positive primary breast cancer. Further testing indicated that the assay might also predict sensitivity to tamoxifen, or firstgeneration adjuvant chemotherapy. This assay is also under evaluation for clinical utility in a large, multicenter, prospective randomized trial
(TailoRx). Whether these multigene predictors of prognosis will have greater utility than Adjuvant!Online remains to be determined. In the meantime, exploratory analyses are ongoing to identify reliable predictors of response to individual drugs and modern combination drug regimens. These are expected to lead to individualized selection of treatment, or personalized medicine.

\section{SYMPOSIUM I}

Prognostic and Risk Factors (Part I)

\section{S2}

Circulating tumor cells as predictors of recurrence in primary breast cancer

W Janni

I Frauenklinik Innenstadt, Klinikum der Ludwig-Maximilians-Universität, München, Germany

Breast Cancer Research 2007, 9(Suppl 1):S2 (doi: 10.1186/bcr1685)

Minimal residual disease (MRD) (that is, isolated tumor cells (ITC) in bone marrow) may be the source of potentially fatal overt distant metastases in solid tumors even years after primary treatment. MRD can be detected by immunohistochemical methods using antibodies directed against cytokeratins, cell-surface markers, or molecular, PCRbased techniques. Among solid tumors, the clinical relevance of MRD has been most extensively studied in breast cancer patients. Recently, the highest level of evidence for the prognostic impact of MRD in primary breast cancer was reached by a pooled analysis comprising more than 4,000 patients, showing poor outcome in patients with MRD at primary therapy. Yet, clinical application of MRD detection is hampered by the lack of a standardized detection assay. Moreover, clinical trial results demonstrating the benefit of a therapeutic interference derived from bone marrow status are still missing. Recent results suggest that, in addition to its prognostic impact, MRD can be used for therapy monitoring or as a potential therapeutic target after phenotyping of the tumor cells. Persisting MRD after primary treatment may lead to an indication for extended adjuvant therapy.

In a pooled analysis, bone marrow aspirates of 726 patients from academic breast cancer units in Oslo $(n=356)$, Munich $(n=228)$ and Tuebingen ( $n=142)$ were analyzed during recurrence-free follow-up at a mean interval of 31.7 months after primary diagnosis of breast cancer pT1-4, pN0-3 pM0. Persistent ITC were detected in $15.4 \%$ of the patients $(n=112)$. The Kaplan-Meier estimate for mean distant relapse-free survival estimate was 163.6 months in patients with negative bone marrow status and 105.2 months in patients with positive bone marrow status. Patients without evidence of persistent ITC had a significantly longer overall survival (165.6 months), than patients with positive bone marrow status ( 103.3 months, $P<0.0001$ ). In multivariate Cox regression analysis, allowing for bone marrow 
status, tumor size, nodal status and histopathological grading, ITC was of independent prognostic significance for subsequent reduced breast cancer specific survival (RR 6.3,95\% Cl 2.3-17.6, $P<0.0001)$, next to nodal status at time of primary diagnosis (RR $2.9,95 \% \mathrm{Cl} 1.8-4.7$ ). Given these inspiring results on ITC in bone marrow, several trials currently analyze the prognostic relevance of circulating tumor cells (CTCs) in peripheral blood in the adjuvant setting. In Germany, for example, CTCs in peripheral blood of breast cancer patients at primary diagnosis and during adjuvant chemotherapy as well as endocrine and bisphosphonate treatment within the SUCCESS Trial $(n=3,658$ patients) are currently analyzed. In this study, the CellSearchSystem (Veridex, Warren, USA) is used for isolation and enumeration of CTCs from $23 \mathrm{ml}$ peripheral blood. Briefly, after immunomagnetic enrichment with an anti-Epcam antibody, cells were labelled with anticytokeratin $(8,18,19)$ and anti-CD45 antibodies to distinguish epithelial cells and leukocytes. In an interim analysis, 456 breast cancer patients at the time of primary diagnosis before the start of systemic adjuvant treatment were analyzed. In this patient group, $28 \%$ of patients $(n=129)$ presented with $\geq 1$ CTC in peripheral blood. In patients with the detection of CTCs, the mean number of cells was four (range 1-166). In 19 healthy individuals we found one CTC in the blood of two patients. The presence of CTCs did not correlate with tumor size $(P=0.18)$, presence of lymph node metastases $(P=0.09)$, histopathological grading $(P=0.56)$, hormone receptor status $(P=0.49)$ or Her2/neu status of the primary tumor $(P=0.33)$.

Persisting MRD after primary treatment may lead to an indication for extended adjuvant therapy. Until clinical consequences of MRD detection in solid tumors and particularly in breast cancer have been validated, however, the detection of isolated tumor cells in bone marrow should be performed mainly in clinical trials.

\section{S3}

\section{Circulating tumor and endothelial cells as predictors of response in metastatic breast cancer M Cristofanilli}

Department of Breast Medical Oncology, The University of Texas MD Anderson Cancer Center, Houston, TX, USA

Breast Cancer Research 2007, 9(Suppl 1):3 (doi: 10.1186/bcr1686)

The detection of microscopic disease is associated with prognostic implications in primary breast cancer [1]. In metastatic breast cancer (MBC), reliable detection of circulating tumor cells (CTCs) has been obtained using immunomagnetic separation and subsequent analysis by the CellSpotter ${ }^{\mathrm{TM}}$ analyzer (Veridex LLC, a Johnson \& Johnson company, Warren, NJ, USA) $[2,3]$. This technology is becoming a standard tool for the 'real-time' assessment of prognosis and response to treatment $[4,5]$. The detection of CTCs in patients with MBC about to start a new line of treatment has been shown to predict progressionfree survival and overall survival and treatment benefit. The prognostic value was independent of the line of therapy (for example, first line versus second line or more) [2,3]. Moreover, in multivariate analysis CTCs demonstrated superior value compared with the site of metastasis (for example, visceral versus soft tissue/bone), type of therapy, and length of time to recurrence after definitive primary surgery. Furthermore, the prognostic value of CTCs has been shown to be superior to standard tumor markers (for example, Ca27-29), tumor burden and phenotype of disease [6]. We have recently focused on determining the feasibility of the genotypic characterization of CTCs and correlating with the expression of similar genes in primary or metastatic lesions [7].

Increases in the number of circulating endothelial cells (CECs) and progenitors (CEPs) have been reported in various pathological conditions including cancer $[8,9]$. At the clinical level, evidence is emerging that CEC kinetics and viability might correlate with clinical outcomes in cancer patients who undergo anti-angiogenic treatment [10]. The CellSpotter ${ }^{\mathrm{TM}}$ analyzer is also being investigated for measurement and characterization of CEC [11]. Therefore, CEC and CEP measurement has potential as a surrogate marker for monitoring antiangiogenic treatment and drug activity, and could help to determine the optimal biological dose of anti-angiogenic drugs, which are being used with increasing frequency in medical oncology.

References

1. Braun $S$, Pantel K, Müller $P$, et al.: Cytokeratin-positive cells in the bone marrow and survival of patients with stage I, II or III breast cancer. N Engl J Med 2000, 342:525-533.

2. Cristofanilli M, Budd GT, Ellis M, et al.: Circulating tumor cells predict progression free survival and overall survival in metastatic breast cancer. N Engl J Med 2004, 351:781-791.

3. Cristofanilli M, Hayes DF, Budd GT, et al.: Circulating tumor cells: a novel prognostic factor for newly diagnosed metastatic breast cancer. J Clin Oncol 2005, 23:1420-1430.

4. Hayes DF, Cristofanilli M, Budd GT, et al.: Circulating tumor cells at each follow-up time point during therapy of metastatic breast cancer patients predict progression-free and overall survival. Clin Cancer Res 2006, 12:4218-4224.

5. Budd GT, Cristofanilli M, Ellis M, et al:: Circulating tumor cells versus imaging - predicting overall survival in metastatic breast cancer. Clin Cancer Res 2006, 12:6403-6409.

6. Cristofanilli M, Kristine Broglio KR, Guarneri V, et al:: Circulating tumor cells in metastatic breast cancer (MBC): biological staging beyond tumor burden. Clin Breast Cancer 2007, 7: 471-479.

7. Reuben JM, Lee B-N, Li C, et al.: Genomic of circulating tumor cells in metastatic breast cancer [abstract]. ASCO 2007, $26:$ in press.

8. Cines DB, et al:: Endothelial cells in physiology and in the pathophysiology of vascular disorders. Blood 1998, 91:35273561.

9. Mancuso $\mathrm{P}$, et al.: Resting and activated endothelial cells are increased in the peripheral blood of cancer patients. Blood 2001, 97:3658-3661.

10. Mancuso $P$, et al.: Circulating endothelial cell kinetics and viability predict survival in breast cancer patients receiving metronomic chemotherapy. Blood 2006, 108:452-459.

11. Smirnov DA, Foulk BW, Doyle GV, et al:: Global gene expression profiling of circulating endothelial cells in patients with metastatic carcinomas. Cancer Res 2006, 66:2918-2922.

\section{SYMPOSIUM I}

\section{Prognostic and Risk Factors (Part II)}

\section{S4}

\section{Gene expression profiling for prognosis of breast cancer}

\section{M van de Vijver}

Department of Pathology, Netherlands Cancer Institute, Amsterdam, The Netherlands

Breast Cancer Research 2007, 9(Suppl 1):S4 (doi: 10.1186/bcr1687)

Tumor factors that can predict prognosis and response to specific therapies (for example, chemotherapy regimens) are of great potential benefit for tailored treatment of patients with invasive breast cancer.

We have previously defined a gene expression profile of 70 genes that is predictive for a short interval to distant metastases ( $<5$ years) in lymph node negative (LNO) patients [1]. We have subsequently validated the prognostic value of this 70-gene profile in a cohort of 295 stage I and II breast cancer patients younger than 53 years of age [2].

To test whether gene expression profiling can be used in clinical practice, we have performed a study in 16 hospitals in The Netherlands. Female patients younger than 61 years presenting with primary operable cT1-2NOMO breast carcinoma were eligible and entered into this prospective feasibility study. Fresh tumor samples in a standardized fashion were collected within 1-3 hours of primary surgery and sent in RNAlater ${ }^{\circledR}$ to the Netherlands Cancer Institute. The 70-gene 
signature (genomic profile) was obtained in node-negative patients with a representative tumor sample; node-positive patients were excluded. Between 2004 and 2006, 812 patients were enrolled and 427 (53\%) genomic profiles were obtained. The remaining $385(47 \%)$ patients were excluded mainly because of node-positive disease (22\%). The logistics of obtaining fresh-frozen material from the tumor has gone very well in each of the participating hospitals. Approximately $50 \%$ of the tumors were shown to have a good prognosis signature.

Another area of research is to identify gene expression signatures associated with the response to chemotherapy. Within a singleinstitution prospective phase II trial, patients with locally advanced breast cancer received six courses of either doxorubicin-cyclophosphamide (AC) $(n=25)$ or doxorubicin-docetaxel (AD) $(n=24)$ containing neoadjuvant chemotherapy. Gene expression profiles were generated from core needle biopsies obtained before treatment and correlated with the response of the primary tumor to the chemotherapy administered [3]. Additionally, pretreatment gene expression profiles were compared with those in tumors remaining after chemotherapy $(n=15)$.

Eleven (22\%) of the 49 patients showed a (near) pathological complete remission of the primary tumor after treatment. No gene expression pattern correlating with response could be identified for all patients as well as for the AC-treated or AD-treated group separately. The comparison of the pretreatment biopsy and the tumor excised after chemotherapy revealed differences in gene expression in those tumors that showed a partial remission but not in tumors that did not respond to chemotherapy.

No gene expression profile predicting the response of primary breast carcinomas to AC-based or AD-based neoadjuvant chemotherapy could be detected in this study. We are currently expanding the series of patients in this neoadjuvant chemotherapy study.

We conclude that gene expression profiling is a method that has greatly accelerated the identification of prognostic and predictive factors in breast cancer and will lead to novel diagnostic tests that can be reliably implicated in clinical practice.

References

1. van 't Veer $L J$, Dai $H$, van de Vijver MJ, et al.: Gene expression profiling predicts clinical outcome of breast cancer. Nature 2002, 415:530-536.

2. van de Vijver M, He Y, Van 't Veer L, Dai H, Hart A, Voskuil D, Schreiber G, Peterse J, Roberts C, Marton M, et al:: A geneexpression signature as a predictor of survival in breast cancer. N Engl J Med 2002, 347:1999-2009.

3. Hannemann J, Oosterkamp HM, Bosch CAJ, Velds A, Wessels LFA, Loo C, Rutgers EJ, Rodenhuis S, van de Vijver MJ: Changes in gene expression associated with response to neoadjuvant chemotherapy in breast cancer. J Clin Oncol 2006, 23:33313342 .

\section{S5}

\section{Molecular and gene expression-based predictors of response to preoperative chemotherapy L Pusztai}

The University of Texas MD Anderson Cancer Center, Houston, TX, USA

Breast Cancer Research 2007, 9(Suppl 1):S5 (doi: 10.1186/bcr1688)

Many single-gene molecular markers have been evaluated as predictors of response to specific regimens. However, no reliable and routinely used molecular chemotherapy response predictors exist today. Molecular markers of proliferative activity remain nonspecific predictors of chemotherapy sensitivity in general. Multidrug-resistance transport proteins, p53 gene mutations, and defects in apoptotic pathways remain highly controversial as predictors of response or resistance to particular drugs. To date, the strongest (although still indirect) evidence supporting a molecular predictor of response to a particular regimen comes from a retrospective subset analysis of a variety of studies that showed a link between topoisomerase II amplification and increased sensitivity to anthracyclines. However, the best methodology for determining amplification of topoisomerase II and the appropriate cut-off value to distinguish between individuals with and without amplification have not been established.

Several small studies provided 'proof-of-principle' that the gene expression profile of cancers that are highly sensitive to chemotherapy is different from that of tumors resistant to treatment. Table 1 (overleaf) presents a summary of the current literature. The most exciting possibility implicit to gene expression profiling-based diagnostic tests is that multiple predictors could be applied to a single data set. It is currently technically feasible to perform gene expression profiling on a single biopsy and assess prognosis using a 76-gene, or 70-gene signature, determine the ER and HER-2 status by measuring receptor mRNA levels, predict endocrine sensitivity among the ER-positive patients and estimate the probability of response chemotherapy.

References

1. Chang JC, Wooten EC, Tsimelzon A, et al:: Gene expression profiling for the prediction of therapeutic response to docetaxel in patients with breast cancer. Lancet 2003, 362:362369.

2. Ayers M, Symmans WF, Stec J, et al.: Gene expression profiles predict complete pathologic response to neoadjuvant paclitaxel and fluorouracil, doxorubicin, and cyclophosphamide chemotherapy in breast cancer. J Clin Oncol 2004, 22:22842293.

3. Iwao-Koizumi K, Matoba R, Ueno N, et al:: Prediction of docetaxel response in human breast cancer by gene expression profiling. J Clin Oncol 2005, 23:422-431.

4. Hannemann J, Oosterkamp HM, Bosch CA, et al:: Changes in gene expression associated with response to neoadjuvant chemotherapy in breast cancer. J Clin Oncol 2005, 23:33313342.

5. Gianni L, Zambetti M, Clark K, et al.: Gene expression profiles in paraffin-embedded core biopsy tissue predict response to chemotherapy in women with locally advanced breast cancer. J Clin Oncol 2005, 23:7265-7277.

6. Folgueira MA, Carraro DM, Brentani $\mathrm{H}$, et al.: Gene expression profile associated with response to doxorubicin-based therapy in breast cancer. Clin Cancer Res 2005, 11:74347443.

7. Dressman HK, Hans C, Bild A, Olson JA, et al.: Gene expression profiles of multiple breast cancer phenotypes and response to neoadjuvant chemotherapy. Clin Cancer Res 2006, 12(3 Pt 1): 819-826.

8. Thuerigen $O$, Schneeweiss A, Toedt G, et al:: Gene expression signature predicting pathologic complete response with gemcitabine, epirubicin, and docetaxel in primary breast cancer. $J$ Clin Oncol 2006, 24:1839-1845.

9. Park S, Shimizu C, Shimoyama T, et al.: Gene expression profiling of ATP-binding cassette (ABC) transporters as a predictor of the pathologic response to neoadjuvant chemotherapy in breast cancer patients. Breast Cancer Res Treat 2006, 99:9-17.

10. Cleator S, Tsimelzon A, Ashworth A, et al.: Gene expression patterns for doxorubicin (Adriamycin) and cyclophosphamide (cytoxan) (AC) response and resistance. Breast Cancer Res Treat 2006, 95:229-233.

11. Hess KR, Anderson K, Symmans WF, et al.: Pharmacogenomic predictor of sensitivity to preoperative chemotherapy with paclitaxel and fluorouracil, doxorubicin, and cyclophosphamide in breast cancer. J Clin Oncol 2006, 24:4236-4244.

12. Mina L, Soule SE, Badve S, et al:: Predicting response to primary chemotherapy: gene expression profiling of paraffinembedded core biopsy tissue. Breast Cancer Res Treat 2006, 103:197-208. 
Table 1 (abstract S5)

\begin{tabular}{|c|c|c|c|}
\hline Reference & $\begin{array}{l}\text { Patient } \\
\text { characteristics }\end{array}$ & Treatment & Results \\
\hline Chang et al. [1] & $\begin{array}{l}24 \text { (discovery) and } \\
6 \text { (validation) } \\
\text { patients. with LABC }\end{array}$ & $\begin{array}{l}4 \text { cycles docetaxel } \\
\text { every } 3 \text { weeks }\end{array}$ & $\begin{array}{l}92 \text { differentially expressed genes }(P<0.001) \\
\text { LOOCV results in training: predictive accuracy of } 88 \%(95 \% \mathrm{Cl}, 68-97 \%) \text {, PPV } \\
\text { of } 92 \%, \mathrm{NPV} \text { of } 83 \% \text {, sensitivity of } 85 \%(95 \% \mathrm{Cl}, 55-98 \%) \text {, specificity of } 91 \% \\
(95 \% \mathrm{Cl}, 59-100 \%) \\
\text { Independent validation: all six patients with sensitive tumors correctly identified }\end{array}$ \\
\hline Ayers et al. [2] & $\begin{array}{l}24 \text { (discovery) and } \\
12 \text { (validation) } \\
\text { patients with breast } \\
\text { cancer }\end{array}$ & $\begin{array}{l}3 \text { or } 12 \text { cycles paclitaxel and } \\
4 \text { cycles of FAC (T/FAC) }\end{array}$ & $\begin{array}{l}\text { No single gene sufficiently associated with pCR to serve as single valid marker } \\
74 \text {-gene predictor for response to chemotherapy } \\
\text { - Predictive accuracy of } 78 \%(95 \% \mathrm{Cl}, 52-94 \%) \text {, PPV of } 100 \% \\
\text { (95\% Cl, } 29-100 \%) \text {, NPV of } 73 \%(95 \% \mathrm{Cl}, 45-92 \%) \text {, sensitivity of } 43 \% \\
(95 \% \mathrm{Cl}, 10-82 \%) \text {, specificity of } 100 \%(95 \% \mathrm{Cl}, 72-100 \%)\end{array}$ \\
\hline $\begin{array}{l}\text { Iwao-Koizumi } \\
\text { et al. [3] }\end{array}$ & $\begin{array}{l}44 \text { (discovery) and } \\
26 \text { (validation) } \\
\text { patients. with } \\
\text { stage II/IV breast canc }\end{array}$ & 4 cycles of docetaxel & $\begin{array}{l}\text { 85-gene-predictor of pCR with: } \\
\text { - Predictive accuracy of } 80.7 \% \text { ( } 95 \% \mathrm{Cl}, 63.5-92.5 \%) \text {, PPV of } 73.3 \% \\
(95 \% \mathrm{Cl}, 49.5-90.3 \%) \text {, NPV of } 90.9 \%(95 \% \mathrm{Cl}, 65.9-99.4 \%) \text {, sensitivity of } \\
91.7 \%(95 \% \mathrm{Cl}, 68.1-99.5 \%) \text {, specificity of } 71.4 \%(95 \% \mathrm{Cl}, 46.7-89.5 \%)\end{array}$ \\
\hline Hannemann et al. [4] & $\begin{array}{l}48 \text { patients with } \\
\text { LABC }\end{array}$ & $\begin{array}{l}6 \text { cycles of doxorubicin/docetaxel } \\
(\mathrm{AD}, n=24) \text { or doxorubicin/ } \\
\text { cyclophosphamide }(\mathrm{AC}, n=24)\end{array}$ & $\begin{array}{l}\text { No differentially expressed genes identified, no possible multigene predictor } \\
\text { developed } \\
\text { Patient samples did not cluster distinctly for pCR/near pCR compared with RD in } \\
\text { hierarchical clustering }\end{array}$ \\
\hline Gianni et al. [5] & $\begin{array}{l}89 \text { (INT-Milan) and } \\
82 \text { (MDACC) } \\
\text { patients with LABC }\end{array}$ & $\begin{array}{l}3 \text { cycles of doxorubicin/paclitaxel, } \\
12 \text { cycles of weekly paclitaxel }\end{array}$ & $\begin{array}{l}86 \text { genes correlating with pCR }(P<0.05) \text { forming three clusters } \\
\text { - ER gene cluster } \\
\text { - Proliferation gene cluster } \\
\text { - Immune-related gene cluster } \\
\text { RS correlated with pCR in INT-Milan patients } \\
86 \text {-gene model developed on INT-Milan patients validated on MDACC samples }\end{array}$ \\
\hline \multirow[t]{2}{*}{ Folgueira et al. [6] } & $\begin{array}{l}\text { (1) } 38 \text { (discovery), } \\
13 \text { (validation) }\end{array}$ & & $\begin{array}{l}\text { (1) } 25(3.8 \%) \text { differentially expressed transcripts between responders and } \\
\text { non-responders, three-gene classifier could not be validated }\end{array}$ \\
\hline & $\begin{array}{l}\text { (2) } 31 \text { (discovery), } \\
13 \text { (validation) }\end{array}$ & & $\begin{array}{l}\text { (2) Three-gene classifier successfully developed and validated both by LOOCV } \\
\text { and in an independent validation set }\end{array}$ \\
\hline Dressman et al. [7] & $\begin{array}{l}37 \text { patients with } \\
\text { stage IIB/III breast } \\
\text { cancer }\end{array}$ & $\begin{array}{l}\text { Liposomal doxorubicin } \times 4 / \text { paclitaxel } \\
\text { combined with local whole breast } \\
\text { hyperthermia }\end{array}$ & $\begin{array}{l}\text { (a) 22-gene signature characterizing IBC identified and validated by LOOVC } \\
\text { (b) 18-gene signature characterizing IBC identified and validated by LOOVC } \\
\text { (c) No gene signature predicting clinical response could be identified }\end{array}$ \\
\hline Thuerigen et al. [8] & $\begin{array}{l}52 \text { (discovery/ } \\
\text { GEsDoc), } 48 \\
\text { (validation/GEDoc) } \\
\text { patients with } \\
\text { T2-4NO-2M0 } \\
\text { breast cancer }\end{array}$ & $\begin{array}{l}\text { GEsDoc (gemcitabine, epirubicin } \\
\text { every } 2 \text { weeks x } 5 \text { followed by } \\
\text { docetaxel every } 2 \text { weeks } \times 2 \text { ), } \\
\text { GEDoc (gemcitabine, epirubicin, } \\
\text { docetaxel every } 3 \text { weeks) }\end{array}$ & $\begin{array}{l}\text { 512-gene-signature predictive of pCR: predictive accuracy of } 88 \% \\
\text { (95\% Cl, 75-95\%), PPV of } 64 \%(95 \% \mathrm{Cl}, 39-81 \%), \mathrm{NPV} \text { of } 95 \% \\
(95 \% \mathrm{Cl}, 82-99 \%) \text {, sensitivity of } 78 \%(95 \% \mathrm{Cl}, 40-97 \%) \text {, specificity of } 90 \% \\
(95 \% \mathrm{Cl}, 76-97 \%)\end{array}$ \\
\hline Park et al. [9] & $\begin{array}{l}21 \text { patients with } \\
\text { stage II/III breast } \\
\text { cancer }\end{array}$ & $\begin{array}{l}4 \text { cycles of FEC every } 3 \text { weeks } \\
\text { followed by } 12 \text { cycles of weekly } \\
\text { paclitaxel }\end{array}$ & $\begin{array}{l}11 \text { differentially expressed } \mathrm{ABC} \text { transporters } \\
\text { Multigene predictor model with the } \mathrm{ABC} \text { transporters differentially expressed } \\
\text { between the two classes }(P<0.003) \text { with predictive of pCR with: } \\
\text { - Predictive accuracy of } 92.8 \%(95 \% \mathrm{Cl}, 88.0-97.4 \%), \mathrm{PPV} \text { of } 93.2 \% \\
(95 \% \mathrm{Cl}, 85.2-100 \%), \mathrm{NPV} \text { of } 93.6 \%(95 \% \mathrm{Cl}, 87.8-99.4 \%) \text {, sensitivity of } \\
88.1 \%(95 \% \mathrm{Cl}, 76.8-99.4 \%) \text {, specificity of } 95.9 \%(95 \% \mathrm{Cl}, 87.8-100 \%)\end{array}$ \\
\hline Cleator et al. [10] & $\begin{array}{l}40 \text { patients with } \\
\text { primary breast } \\
\text { cancer }\end{array}$ & $\begin{array}{l}6 \text { cycles doxorubicin/ } \\
\text { cyclophosphamide (AC) }\end{array}$ & $\begin{array}{l}253 \text { differentially expressed genes; } \\
-75 \text { genes overexpressed in resistant tumors (that is, transcription, differentiation, } \\
\text { signal transduction, amino acid metabolism) } \\
-178 \text { genes overexpressed in sensitive tumors (that is, cell cycle, survival, stress } \\
\text { response, and estrogen-regulated genes) }\end{array}$ \\
\hline Hess et al. [11] & $\begin{array}{l}82 \text { (discovery), } \\
51 \text { (validation) }\end{array}$ & $\begin{array}{l}3 \text { or } 12 \text { cycles paclitaxel and } \\
4 \text { cycles of FAC (T/FAC) }\end{array}$ & $\begin{array}{l}30-\text { gene predictor identified and validated by fivefold cross-validation, permutation } \\
\text { testing and application to an independent data set: } \\
\text { - Predictive accuracy of } 76 \%(95 \% \mathrm{Cl}, 62-87 \%), \mathrm{PPV} \text { of } 52 \% \\
(95 \% \mathrm{Cl}, 31-73 \%) \text {, NPV of } 96 \%(95 \% \mathrm{Cl}, 82-100 \%) \text {, sensitivity of } 92 \% \\
(95 \% \mathrm{Cl}, 64-100 \%) \text {, specificity of } 71 \%(95 \% \mathrm{Cl}, 54-85 \%)\end{array}$ \\
\hline Mina et al. [12] & $\begin{array}{l}45 \text { patients with } \\
\text { stage II/III breast } \\
\text { cancer }\end{array}$ & $\begin{array}{l}3 \text { cycles doxorubicin every } \\
2 \text { weeks and } 6 \text { cycles docetaxel } \\
\text { weekly } x 6\end{array}$ & $\begin{array}{l}22 \text { of } 274 \text { candidate genes correlated with } \mathrm{pCR}(P<0.05) \text { forming three large } \\
\text { clusters: angiogenesis-related genes, proliferation-related genes, invasion-related } \\
\text { genes, no correlation between RS and pCR, } 24 / 274 \text { genes correlated with } \\
\text { inflammatory phenotype }\end{array}$ \\
\hline
\end{tabular}

ABC transporter, ATP binding cassette transporter; discovery, development/training set; FAC, 5-fluorouracil, doxorubicine, cyclophosphamide; FEC, 5-fluorouracil, epirubicin, cyclophosphamide; IBC, inflammatory breast cancer; LABC, locally advanced breast cancer; LOOCV, leave-one-out cross-validation; NPV, negative predictive 


\section{S6}

\section{Role of MR and digital mammography for screening DD Dershaw}

Memorial Sloan-Kettering Cancer Center, New York, NY, USA

Breast Cancer Research 2007, 9(Suppl 1):S6 (doi: 10.1186/bcr1689)

New technologies have raised the issue of their application as a replacement for or in addition to screen-film mammography in screening of women at normal risk and elevated risk of breast cancer.

Digital mammography images the breast using the identical information obtained in screen-film mammography. The image is processed, stored and displayed electronically. This conveys several advantages over film techniques, but the approval of digital mammography by the US Food and Drug Administration has been based on comparable ability to detect cancer, not any diagnostic advantage.

Four prospective studies comparing digital and film mammography on the same patients have shown that for population-based screening there is no advantage for digital over film. The last and largest of these studies [1] initially reported an advantage for several subgroups of women for digital screening. Later analysis of data from this study, however, concluded that only women with dense breasts may benefit and that screening of entire populations with digital mammography is excessively costly and not beneficial.

Analysis of MR as a screening tool has been directed at women with greatly elevated lifetime risk of developing breast cancer. The ability of MR to detect a large percentage of cancers in these women earlier than mammography, sonography or physical examination and at a stage at which they should be curable has been clearly demonstrated. This has lead the American Cancer Society, along with others, to recommend the use of MR to annually screen women with at least a $20 \%$ lifetime risk of developing breast cancer starting at age 25 years. Those at less risk were not included due to lack of supporting data and concern over excessive biopsies in those women.

Reference

1. Pisano ED, Gatsonis C, Hendrick E, et al.: Diagnostic performance of digital versus film mammography for breast-cancer screening. N Engl J Med 2005, 353:1773-1783.

\section{SYMPOSIUM II}

\section{New Strategies for Primary Treatment (Part I)}

\section{S7}

\section{Surgical issues in the breast and axillary nodes in patients treated with neoadjuvant systemic therapy EP Mamounas ${ }^{1,2}$}

${ }^{1}$ Aultman Cancer Center, Canton, OH, USA; ${ }^{2}$ Northeastern Ohio Universities College of Medicine, Rootstown, $\mathrm{OH}, \mathrm{USA}$

Breast Cancer Research 2007, 9(Suppl 1):S7 (doi: 10.1186/bcr1690) Several unique surgical issues arise in the management of patients who are candidates for neoadjuvant systemic therapy. These involve the original diagnostic assessment of the extent of disease in the breast and axilla, the preoperative planning, and ultimately the surgical management of the primary breast tumor and that of axillary lymph nodes. Careful consideration of these issues is crucial in order to maximize local control of the disease, while minimizing the extent of the required surgical resection and the ensuing surgical morbidity.

Adequate diagnostic assessment with core needle biopsy before the initiation of neoadjuvant systemic therapy ensures the presence of invasive carcinoma and provides adequate material for routine biomarker evaluation (such as ER, PR and HER-2 neu), while minimally disturbing the primary breast tumor. Consideration should also be given in assessing the status of axillary nodes by minimally invasive techniques such as ultrasound of the axilla and fine needle aspiration of suspicious nodes. Optimal preoperative planning aims at accurately determining the patterns of primary tumor shrinkage and the amount and location of any residual disease in the breast.

Surgical treatment after neoadjuvant systemic therapy focuses on the management of the primary breast tumor and that of axillary lymph nodes. Regarding the primary breast tumor, several studies have shown that neoadjuvant systemic therapy converts a proportion of mastectomy candidates to candidates for breast-conserving surgery. Neoadjuvant systemic therapy can also decrease the amount of breast tissue that needs to be removed at lumpectomy even in patients who are lumpectomy candidates at presentation.

Neoadjuvant systemic therapy (primarily neoadjuvant chemotherapy) downstages axillary lymph nodes in up to $30-40 \%$ of the patients. Although this observation was of little clinical significance when axillary node dissection was the sole method for staging the axilla, the development and validation of sentinel lymph node biopsy has provided an additional potential advantage for neoadjuvant chemotherapy; that is, the possibility of decreasing the extent and morbidity of axillary surgery. This approach is, naturally, predicated on the premise that sentinel node biopsy is feasible and accurate following neoadjuvant chemotherapy. Until recently, only small, single-institution studies have examined the efficacy of lymphatic mapping and the accuracy of sentinel node biopsy after neoadjuvant chemotherapy with significant variability in the rate of sentinel node identification and in the rate of false negative sentinel node [1]. When these studies are examined collectively [1,2] or when larger, multicenter data sets are analyzed [3], however, sentinel node biopsy after neoadjuvant chemotherapy appears to have similar performance characteristics to those of sentinel node biopsy before systemic therapy [4-6].

Some have proposed that candidates for neoadjuvant systemic therapy should have a sentinel node biopsy before, rather than after, neoadjuvant systemic therapy so that information on the status of the axillary nodes is obtained without the potential confounding effects of systemic treatment, and sentinel node-negative patients can avoid axillary dissection [7-9]. Although this approach may be useful in patients who will not need systemic therapy (that is, chemotherapy) if the sentinel node is negative, it is not generally useful for the majority of candidates for neoadjuvant systemic therapy, for whom little - if anything - is to be gained by knowing the pathologic nodal status upfront. In addition, this approach commits patients to two surgical procedures and does not take advantage of the down-staging effect of neoadjuvant chemotherapy on the axillary nodes.

\section{References}

1. Mamounas EP: Sentinel lymph node biopsy after neoadjuvant systemic therapy. Surg Clin North Am 2003, 83:931-942.

2. Xing $Y$, Ding D, Cox D, et al.: Meta-analysis of sentinel lymph node biopsy following preoperative chemotherapy in patients with operable breast cancer [abstract 561]. Proc Am Soc Clin Oncol 2004, 23:17.

3. Mamounas EP, Brown A, Anderson S, et al.: Sentinel node biopsy after neoadjuvant chemotherapy in breast cancer: results from National Surgical Adjuvant Breast and Bowel Project Protocol B-27. J Clin Oncol 2005, 23:2694-2702.

4. Veronesi U, Paganelli G, Viale G, et al.: A randomized comparison of sentinel-node biopsy with routine axillary dissection in breast cancer. N Engl J Med 2003, 349:546-553.

5. McMasters KM, Tuttle TM, Carlson DJ, et al:: Sentinel lymph node biopsy for breast cancer: a suitable alternative to routine axillary dissection in multi-institutional practice when optimal technique is used. $J$ Clin Oncol 2000, 18:2560-2566.

6. Krag $\mathrm{D}$, Weaver $\mathrm{D}$, Ashikaga $\mathrm{T}$, et al.: The sentinel node in breast cancer - a multicenter validation study. $N$ Engl J Med 1998, 339:941-946.

7. Bedrosian I, Reynolds C, Mick R, et al.: Accuracy of sentinel lymph node biopsy in patients with large primary breast tumors. Cancer 2000, 88:2540-2545.

8. Schrenk P, Hochreiner G, Fridrik M, et al:: Sentinel node biopsy performed before preoperative chemotherapy for axillary lymph node staging in breast cancer. Breast $J$ 2003, 9:282-287.

9. Sabel MS, Schott AF, Kleer CG, et al.: Sentinel node biopsy prior to neoadjuvant chemotherapy. Am J Surg 2003, 186:102-105. 


\section{S8}

\section{Treatment of the intact primary in women with metastatic breast cancer \\ SA Khan 1,2, H Hazard', D Scholtens ${ }^{2,3}$}

${ }^{1}$ Department of Surgery, ${ }^{2}$ Robert H. Lurie Comprehensive Cancer Center and ${ }^{3}$ Department of Preventive Medicine, Feinberg School of Medicine of Northwestern University, Chicago, IL, USA

Breast Cancer Research 2007, 9(Suppl 1):S8 (doi: 10.1186/bcr1691)

Background Approximately 12,000 women with primary breast cancer present with stage IV disease annually in the United States. The present treatment paradigm is that systemic therapy is the standard of care and surgical therapy for the primary tumor is provided only to palliate symptoms. Methods We have examined the impact of local therapy on survival in patients presenting with stage IV breast cancer at initial diagnosis, reporting to the National Cancer Database (NCDB) between 1990 and 1993. In a subsequent study, we reviewed the medical records of women presenting with stage IV breast cancer to Northwestern Memorial Hospital (NMH) over the 10-year period 1995-2005. Cox regression models and logistic regression was used to estimate adjusted overall survival in both studies, and the time to first progression (TTFP) and chest wall status in the NMH population.

Results A total of 16,024 patients with stage IV disease were identified in the NCDB over 4 years, of whom $42.8 \%$ received only diagnostic or palliative procedures, and $57.2 \%$ underwent partial or total mastectomy. A multivariate proportional hazards model identified the number of metastatic sites, the type of metastatic burden, and the extent of resection of the primary tumor as significant independent prognostic covariates. Women treated with surgical resection with free margins, when compared with those not surgically treated, had superior prognosis, with a hazard ratio of 0.61 (95\% Cl 0.58-0.65). In the $\mathrm{NMH}$ study, we identified 114 women; 48 (42\%) underwent resection of the primary tumor. Local control of the chest wall was maintained in 36/48 (75\%) of the surgical group, versus 31/66 (47\%) patients without surgical therapy $(P=0.002)$. TTFP was prolonged in the surgical group, adjusted HR $0.639(P=0.03)$. The HR for overall survival in the surgical group was $0.724(P=0.160)$. Notably, in women with controlled chest walls we observed an overall survival benefit with a HR of $0.418(P<0.0002)$.

Conclusion Recent retrospective studies suggest that surgical resection of the primary tumor in women who also have distant disease may be beneficial [1-3]. These are remarkably consistent in the magnitude of the survival advantage, with a hazard ratio of about 0.6 when surgical intervention is used. There is a paucity of data supporting the assumption that surgical resection will maintain a disease-free chest wall. A study of women with in-breast recurrence of breast cancer and synchronous distant metastases found that women undergoing resection were more likely to avoid uncontrolled chest-wall disease and to survive longer [4]. Our study at $\mathrm{NMH}$ (the largest reported to date examining this issue) finds that maintenance of a disease-free chest wall is associated with improved survival. This further emphasizes the need for prospective randomized trials to establish the role of local therapy in the setting of metastatic breast cancer.

References

1. Khan SA, Stewart AK, Morrow M: Does aggressive local therapy improve survival in metastatic breast cancer? Surgery 2002, 132:620-626.

2. Rapiti E, Verkooijen HM, Vlastos G, Fioretta G, Neyroud-Caspar I, Sappino AP, Chappuis PO, Bouchardy C: Complete excision of primary breast tumor improves survival of patients with metastatic breast cancer at diagnosis. J Clin Oncol 2006, 24: 2743-2749.

3. Babiera GV, Rao R, Feng L, Meric-Bernstam F, Kuerer HM, Singletary SE, Hunt KK, Ross MI, Gwyn KM, Feig BW, Ames FC, Hortobagyi GN: Effect of primary tumor extirpation in breast cancer patients who present with stage IV disease and an intact primary tumor. Ann Surg Oncol 2006, 13:776-782.

4. Dalberg K, Liedberg A, Johansson U, Rutqvist LE: Uncontrolled local disease after salvage treatment for ipsilateral breast tumour recurrence. Eur J Surg Oncol 2003, 29:143-154.

\section{S9}

\section{Immediate versus delayed breast reconstruction GL Robb}

Division of Plastic Surgery, Department of Surgery, The University of Texas MD Anderson Cancer Center, Houston, TX, USA

Breast Cancer Research 2007, 9(Suppl 1):S9 (doi: 10.1186/bcr1692)

With numerous advances in the field of plastic surgery, breast reconstruction is available today to almost any woman undergoing surgery for breast cancer. Several methods can be used for restoration of the breast either at the same time as breast cancer surgery (immediate reconstruction) or months or even years later, at the patient's discretion (delayed reconstruction). The skin sparing approach to mastectomy, originated in the early 1990s, has especially facilitated immediate reconstruction of the breast and resulted in a high standard of cosmetic outcomes. In general, the reconstructive options include using autologous soft tissues from the patient's donor areas, such as the abdomen, hips, back, or buttock, or using a prosthetic implant to create a new breast mound. For well over a decade now, skin-sparing mastectomy and immediate reconstruction has offered the compelling advantages of both superior cosmetic appearance of the reconstructed breast as well as favorable psychological and economic benefits [1].

For many years in the past there was the misconception that breast reconstruction must be delayed for several years after mastectomy because reconstruction might prevent or delay detection of local recurrence. Hence, a large population of women has sought and presently still seeks to undergo delayed postmastectomy reconstruction months to years later. The outcomes of these reconstructions are good to excellent but the cosmetic outcome is always somewhat inferior to the results obtainable by immediate reconstruction because of the loss of the breast skin envelope after mastectomy and the need to replace so much of the chest wall skin. In regard to the all-important question of local recurrence, several longer term follow-up studies at the MD Anderson Cancer Center actually indicate a lower incidence of local recurrence after immediate reconstruction.

Current comparative statistics for the numbers of patients undergoing immediate as well as delayed reconstruction from 2002-2006 are presented in Table 1. Since 2002, the MD Anderson Plastic Surgery Department has seen a progressively increasing demand of early breast cancer patients for implant-based reconstructions performed on an immediate basis. The opposite trend of a preference of autologous tissue reconstructions is apparent for delayed reconstructions.

The controversy today is that we continue to undergo an important evolution in our approach to breast reconstruction because of the identified advantage of the addition of postmastectomy radiation therapy to mastectomy and chemotherapy. The inability to determine which patients will require postmastectomy radiation therapy, especially which patients with early-stage breast cancer, has increased the complexity of planning for immediate breast reconstruction. There are two potential problems with performing an immediate breast reconstruction in a patient who will require postmastectomy radiation therapy. One problem is that postmastectomy radiation therapy can adversely affect the aesthetic outcome of an immediate breast reconstruction [1-3]. The other potential problem is that an immediate breast reconstruction can interfere with the delivery of postmastectomy radiation therapy. Because radiation therapy is one of the most important considerations affecting the timing and technique of breast reconstruction, plastic surgeons and radiation oncologists must work together to maintain an appropriate balance between minimizing the risk of recurrence and providing the best possible aesthetic outcome. Our experience at the MD Anderson Cancer Center and many of the other experiences reported in the literature indicate that breast reconstruction should probably be delayed in patients who are known preoperatively to require postmastectomy radiation therapy $[2,4]$.

Considering, then, our evolving approach for early breast cancer patients who want immediate breast reconstruction but are at higher risk for postmastectomy radiation therapy and likely delayed reconstruction, our updated 'delayed-immediate' [5] reconstruction experience between May 2002 and November 2006 includes 42 


\begin{tabular}{|c|c|c|c|c|c|c|}
\hline \multirow[b]{2}{*}{ Year } & \multicolumn{2}{|c|}{ Number of immediate reconstructions } & \multicolumn{2}{|c|}{ Number of delayed reconstructions } & \multirow{2}{*}{$\begin{array}{l}\text { Total number of } \\
\text { reconstructions }\end{array}$} & \multirow{2}{*}{$\begin{array}{c}\text { Number of } \\
\text { mastectomies }\end{array}$} \\
\hline & Implant & Patient's own tissue & Implant & Patient's own tissue & & \\
\hline 2002 & 167 & 153 & 43 & 55 & 418 & 418 \\
\hline 2003 & 186 & 176 & 53 & 68 & 483 & 482 \\
\hline 2004 & 256 & 158 & 45 & 84 & 543 & 541 \\
\hline 2005 & 239 & 164 & 48 & 75 & 526 & 523 \\
\hline 2006 & 313 & 234 & 39 & 98 & 684 & 683 \\
\hline
\end{tabular}

patients who were considered preoperatively to be at an increased risk of requiring postmastectomy radiation therapy (PMRT) and underwent delayed-immediate breast reconstruction at the MD Anderson Cancer Center. After review of the permanent pathology, 62\% (26 of 42 patients) of patients did not require PMRT and, because of the preserved breast envelope, could go ahead with essentially an immediate reconstruction. The remaining $38 \%$ (16 of 42 patients) did require PMRT, but selected patients are being managed on a similar IRB reconstruction protocol that will allow a 'skin-preserving' delayed reconstruction following completion of the PMRT. Fifteen of these 16 patients (94\%) were evaluated as having uncompromised radiation delivery. Cosmetic results of this approach are very promising and compare favorably with immediate reconstruction in the short-term follow-up to date. Complication rates with delayed-immediate reconstruction are considered quite acceptable and included: stage I (three of 42 patients, 7\%), stage II (four of 26 patients, 15\%), skinpreserving delayed reconstruction (one of 13 patients, 7\%), and expander loss (surgery-related, 2\%; PMRT-related, 6\%). Only 2\% (one of 42 patients) of the patients developed a recurrence of breast cancer after an average follow-up of 24 months.

Breast reconstruction plays an important role in the multidisciplinary, comprehensive care of the breast cancer patient. Advances in techniques have allowed immediate reconstruction to minimize incisional scars on the breast and improve overall breast contour, shape, and appearance. The improved aesthetic outcomes over delayed reconstruction, achieved also by the skin-preserving 'delayed- immediate' approach for early breast cancer patients at risk for radiation therapy and similarly for selected patients actually undergoing PMRT, has convinced many breast cancer patients to view mastectomy with reconstruction as a viable and positive treatment choice. Those patients who are candidates for and who will undergo delayed reconstruction, either because of the planned need for adjuvant radiotherapy or because of poorly controlled comorbidities that must be stabilized before a reconstruction can be undertaken, can still anticipate an excellent cosmetic outcome with a record of high patient satisfaction.

References

1. Robb GL: Reconstructive surgery. In Breast Cancer. MD Anderson Cancer Care Series. Edited by Hunt KK, Strom EA, Ueno NT. New York: Springer-Verlag, Inc.; 2001:223-253.

2. Tran NV, Evans GRD, Kroll SS, Baldwin BJ, Miller MJ, Reece GP, Robb GL: Postoperative adjuvant irradiation: effects on transverse rectus abdominis muscle flap breast reconstruction. Plast Reconstr Surg 2000, 106:313-317.

3. Kronowitz SJ, Robb GL: Breast reconstruction and radiation therapy. In Advanced Therapy of Breast Disease. 2nd edition. Edited by Singletary SE, Robb GL, Hortobagyi GN. London: BC Decker, Inc.; 2003:427-438.

4. Kronowitz SJ, Robb GL: Breast reconstruction with postmastectomy radiation therapy: current issues. Plast Reconstr Surg 2004, 114:950-960.

5. Kronowitz SJ, Hunt KK, Kuerer HM, Babiera G, McNeese MD, Buchholz TA, Strom EA, Robb GL: Delayed-immediate breast reconstruction. Plast Reconstr Surg 2004, 113:1617-1628.

\section{SYMPOSIUM II}

\section{New Strategies for Primary Treatment (Part II)}

\section{S10}

The nipple sparing mastectomy: a 5-year experience at the European Institute of Oncology of Milan JY Petit', U Veronesi ${ }^{2}$, R Orecchia ${ }^{3}$, A Luini ${ }^{4}$

${ }^{1}$ Department of Plastic Surgery, ${ }^{2}$ Scientific Director, ${ }^{3}$ Department of Radiotherapy and ${ }^{4}$ Department of Senology, European Institute of Oncology, Milan, Italy

Breast Cancer Research 2007, 9(Suppl 1):S10 (doi: 10.1186/bcr1693)

Background Under certain conditions, the nipple sparing mastectomy can be proposed for the treatment of breast cancer when the mastectomy remains indicated. The nipple areola complex (NAC) preservation improves the quality of life, reducing the feeling of mutilation. The main argument against the procedure is the lack of radical resection of the retroareolar ductal system. A novel radiosurgical treatment combining subcutaneous mastectomy with intraoperative radiotherapy is proposed.

Methods Seven hundred and seventy-three nipple sparing mastectomies have been performed since March 2002, for invasive carcinoma in $63 \%$ and for in situ carcinoma in $37 \%$. Clinical complications, aesthetic results, and oncological and psychological results were recorded.

Results The NAC necrosed totally in 26 cases (3.3\%) and partially in 49 cases $(6.3 \%)$, and was removed in 36 cases $(4.6 \%)$. Thirteen infections (1.6\%) were observed and $32(4.14 \%)$ prostheses removed. The median rating of the patients for global cosmetic results on a 0 (worst) to 10 (excellent) scale was 8 . The surgeon in charge of the follow-up has given the same rating. A radio dystrophy was observed on the areola in $5.1 \%$ of the patients. The sensitivity of the NAC recovered slowly and partially in only $30 \%$ of the cases. Twelve local recurrences (1.5\%) occurred in an average follow-up period of 18 months (range 1-61 months). Most recurrences were located far from the nipple areola site, outside the radiated field. Two recurrences were Paget disease associated with an in situ carcinoma. Overall, we observed 23 metastases and three deaths. Sixty-eight per cent of patients were satisfied with their reconstructed breast and 97\% were satisfied with having preserved the NAC.

\section{Introduction}

Conservative treatment is now well accepted for a majority of breast cancers and is performed in around $70-80 \%$ of cases [1]. However, mastectomy remains indicated in at least $20 \%$ of patients. An important improvement resulted from the skin-sparing mastectomy technique, validated by several publications [2-11]. The preservation of the skin envelope has favoured the quality of the breast reconstruction, and breast reconstructive surgery has made important progress, providing more natural shape. However, despite the reconstruction, patients often deplore a feeling of mutilation. The nipple areola complex (NAC) 


\section{Figure 1 (abstract S10)}

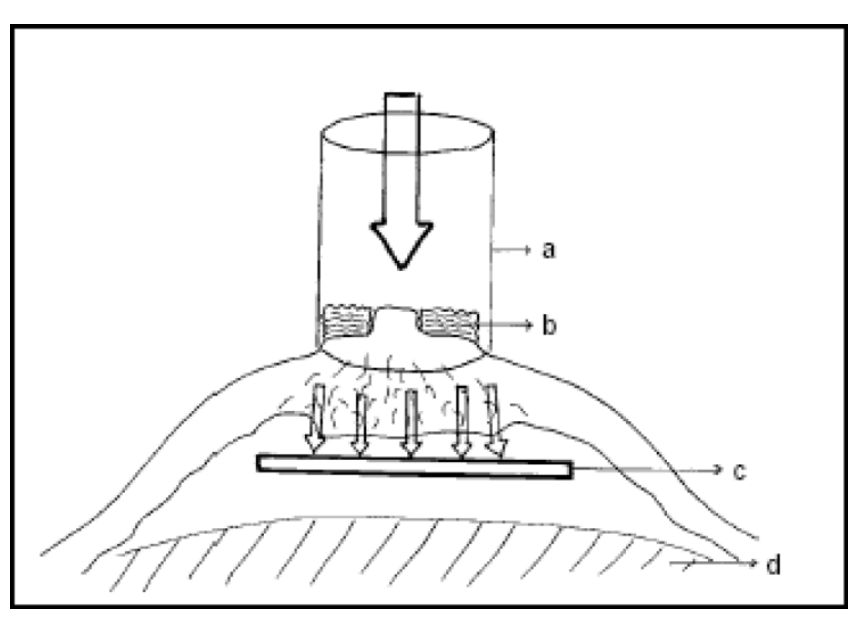

(a) The sterile collimator of the LINAC. (b) The gauze over the areola allows a more homogeneous distribution of the dose to the nipple and the areola. (c) Protective lead and aluminum disks. (d) Pectoralis major muscle.

appears an identity mark of the breast [12]. Several authors have evaluated the risk of nipple areola involvement and investigated the possibility of nipple areola preservation [13-18]. Focusing on such risk, and the possible cancer recurrence in the breast tissue preserved beneath the NAC for the blood supply, we proposed to combine the subcutaneous mastectomy technique with intraoperative radiotherapy with electrons (ELIOT), previously trialled in breast-conserving treatment [19]. The preliminary results of the 'nipple sparing mastectomy' (NSM) were published in 2003 [20]. Today, we report the results of 773 NSMs performed at the European Institute of Oncology.

\section{Materials and methods}

From March 2002 to March 2007, 898 patients were invited to undergo a NSM. The inclusion criteria were primary tumours located at least $1 \mathrm{~cm}$ outside the areola margins, an absence of nipple retraction or bloody discharge, and an absence of retro areola microcalcifications. Multifocality was not a cause for exclusion, provided that all the tumour sites were distant from the areola. Invasive as well as in situ carcinomas were included. Patients were excluded at the time of the operation when the frozen examination of the retro areola tissue had been positive for carcinoma: in these cases, ELIOT was not delivered and the NAC was removed. Among the 898 cases, 98 were excluded, mainly because of the positive extemporaneous examination and in several cases because of the poor blood supply requiring the NAC removal. Fifty-one candidates were excluded because no radiotherapy was delivered, most often when the blood supply was poor and the risk of necrosis very high. Finally, 749 patients underwent a NSM, among whom 24 had a bilateral NSM.

The mean patient age was 46 years (range $20-73$ years). Seventy-five per cent of the patients have a mean follow-up of 18 months (range $1-61$ months).

The surgical technique has already been described [20]. Breast reconstruction was achieved with a definitive prosthesis in 689 cases $(89 \%)$ and with an expander in $185(24 \%)$. Only six patients underwent a reconstruction with an autologous transverse rectus abdominis myocutaneous and one with an autologous Latissimus dorsi.

Once the glandular tissue has been removed and the frozen section of the retro areola tissue proved free of cancer, ELIOT was performed on the NAC area. The ELIOT technique has already been described [21-24]. In our technique, 16 Gy were delivered on the NAC in one shot with lead and aluminium disk protection of the pectoralis muscle and thoracic wall (see Figure 1). However, in 145 cases, the same radiotherapy was postponed to the following day, due to technical problems with the machine in the operating room.

Sensitivity, colour, radio dystrophy, position and symmetry, and global evaluation by the surgeon and the patient, were rated according to a scale $(0-10), 10$ indicating the best results. (The results were rated poor when rated from 0 to 3 , fair from 5 to 6 and good or excellent from 7 to 10.)

Local recurrences, distant metastases and death were also recorded.

A psychological study was conducted concomitantly. A detailed questionnaire was sent to the patients 1 year after the operation to evaluate the degree of satisfaction. The statistic analysis of psychological results was performed with the chi-square test and exact Fisher test.

Results

NAC total necrosis was observed in 26 cases out of 773 NSMs (3.3\%). Partial necrosis was observed in 49 cases (6.3\%). The NAC was removed in 36 cases (4.6\%). The definitive histology of the 773 NSMs was invasive carcinoma in $63 \%$ and in situ carcinoma in $37 \%$. In 53 cases (6\%), the definitive histology of the retro areola tissue returned positive for carcinoma (invasive in 35 cases and in situ in 18 cases). Thirteen infections (1.6\%) were observed in the immediate postoperative period; 32 prostheses were removed (4.1\%). A capsulotomy has been required in 137 cases (17.7\%).

Cosmetic results and sequelea were evaluated by the surgeon in charge of the follow-up according to the scale previously mentioned. The average evaluation of the sensitivity of the areola and the periareolar area was $2 / 10$. Thirty per cent of the patients recovered some kind of sensitivity in several months. The colour of the areola was judged $8 / 10$. The radio dystrophy was absent in most cases: $9 / 10$. A radio dystrophy such as telangiectasia has been observed in $5.1 \%$ of the cases. The symmetry of the breasts was evaluated well in most

\section{Figure 2 (abstract S10)}

(a)

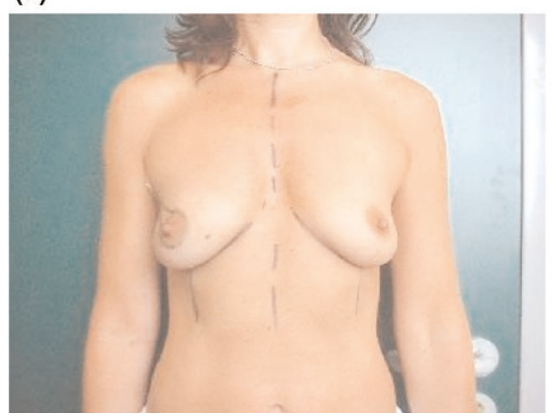

(b)

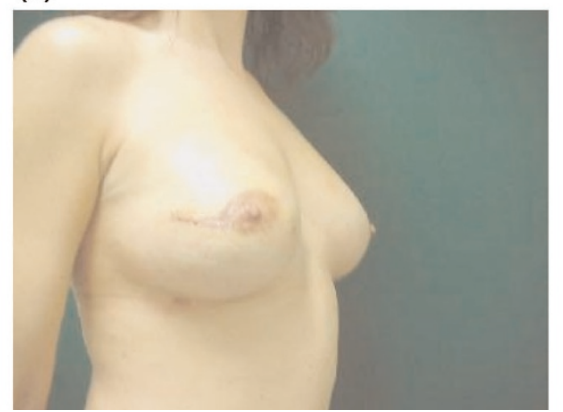

(c)

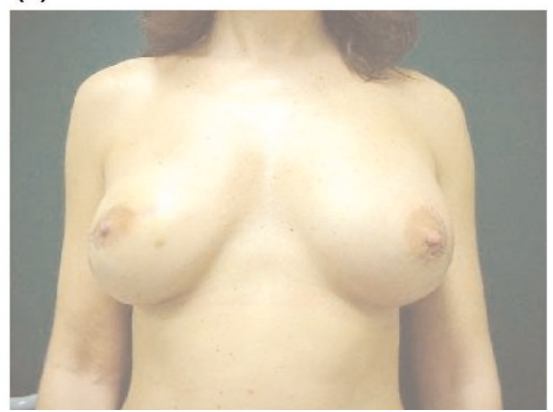

Case 1. (a) Preoperative drawing and result. (b) and (c) Final result with contralateral augmentation. 


\section{Figure 3 (abstract S10)}

(a)

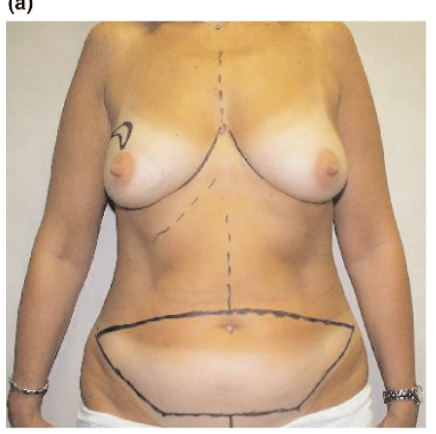

(b)

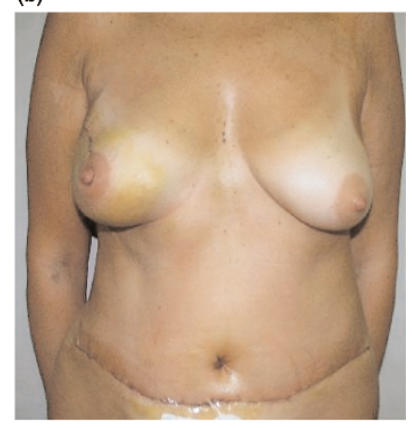

Case 2. (a) Right NSM. (b) Breast reconstruction with TRAM flap.

cases: $7 / 10$. The global result was rated by the patient as $8 / 10$ and also as $8 / 10$ by the surgeon (see cases $1-3$, Figures $2-4$ ).

Cancer results

Despite the frozen section performed underneath the NAC to eliminate the cases with positive results, the final histology of the retro areola tissue was positive in $53(6.8 \%)$ cases, among which 35 were invasive carcinomas (66\%). However, the NAC has been preserved in most cases when the final examination returned positive, taking into account the intraoperative radiotherapy to avoid recurrences. The secondary removal was due to local necrosis. Twelve local recurrences were observed. Two were located on the NAC and the 10 others at a distance from the radiated area, usually in the same area of the tumour location. Both recurrences on the NAC were a DCIS associated with a Paget disease on the nipple. No recurrence on the NAC was observed in the group of patients with persistent positive histology.

Twenty-three cases of distant metastases and three deaths were observed in our series.

The psychological study was completed for 159 patients who had at least 1 year follow-up and who answered our questionnaire. Ninetyseven per cent of the patients interviewed declare being very satisfied with the appearance of the breast after the NSM. No patient regretted having undergone reconstructive surgery, and $91.5 \%$ agreed with the mutilation being decreased by having preserved the NAC. Similarly, $93 \%$ of women responded that conserving the nipple aided in facing illness, and only $1.6 \%$ expressed a total dissatisfaction.

Discussion

Our study on 773 skin-sparing mastectomies performed at the European Institute of Oncology confirms the feasibility of the procedure, with a majority of good results after the preservation of the NAC. However, a partial or total NAC necrosis due to insufficient blood supply was observed in $9.7 \%$ of the cases. Moreover, $6.6 \%$ of the final retro areola histology return positive while it was observed negative at the frozen section. Such results could be improved with a better selection of the NSM, if we cancel the cases requiring an extensive retro areola tissue removal and if we change the surgical indications of the reconstruction in cases of large breast, more often using autologous tissue reconstruction. Concerning the retro areola free margins, we should verify with more accuracy the clinical proximity between the tumour and the NAC.

A partial or global return of the NAC sensitivity was present in only $30 \%$ of the patients. As expected, the return is incomplete and takes months to happen [25]. The risk of radio dystrophy is low with such intraoperative radiotherapy at the level of 16 Gy. Seven per cent of the patients had a severe or moderate radio dystrophy, but a mild pigmentation was observed in around one-third of the women with at least 1 year follow-up.

Two in situ local recurrences were observed on the preserved NAC, which is reassuring although the median follow-up is only 18 months. A

\section{Figure 4 (abstract S10)}

(a)

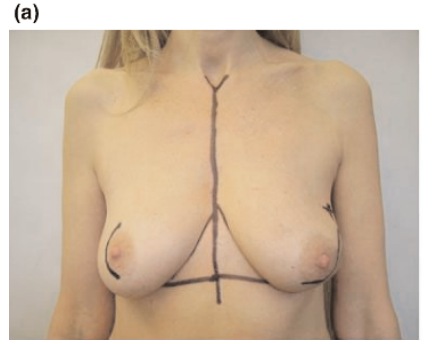

(b)

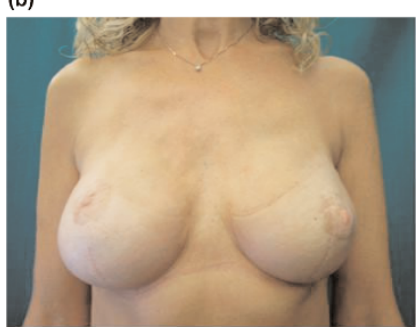

Case 3. (a) Left nipple sparing mastectomy, right prophylactic mastectomy. (b) Bilateral reconstruction with prosthesis.

longer follow-up is required to prove the efficacy of the intraoperative radiotherapy. The dose of ELIOT chosen to reduce the recurrence risk could be questioned [20-24]. The single application of $16 \mathrm{~Gy}$ corresponds to the classic fractioned radiotherapy of $45 \mathrm{~Gy}$ for tumour cells and of $70-80$ Gy for late responding normal tissue. This dose should be sufficient to sterilise more than $90 \%$ of the residual cancer cells, and to obtain an acceptable risk (less than $5 \%$ ) of severe late complications (necrosis). However, the percentage of patients with positive persistent carcinoma behind the NAC in our series justifies ELIOT, although other authors advocate the possibility of NAC preservation without ELIOT [13,26-29].

Finally, the primary goal of the NSM is the psychological improvement of patients requiring a mastectomy. The preliminary results of the questionnaire report a high level of satisfaction and confirm the psychological importance of NAC conservation.

\section{References}

1. Veronesi U, Volterrani F, Luini A, et al:: Quadrantectomy versus lumpectomy for small size breast cancer. Eur J Cancer 1990, 26:671-673.

2. Carlson GW, Losken A, Moore B, et al:: Results of immediate breast reconstruction after skin-sparing mastectomy. Ann Plast Surg 2001, 46:222-228.

3. Carlson GW, Bostwick J, Styblo TM, et al:: Skin-sparing mastectomy. Oncologic and reconstructive considerations. Ann Surg 1997, 225:570-575.

4. Peyser PM, Abel JA, Straker VF, et al.: Ultra conservative skinsparing 'keyhole' mastectomy and immediate breast and areola reconstruction. Ann R Coll Surg Eng/ 2000, 82:227-235.

5. Ho CM, Mak CK, Lau Y, et al:: Skin involvement in invasive breast carcinoma: safety of skin sparing-mastectomy. Ann Surg Oncol 2003, 10:102-107.

6. Carlson GW, Styblo TM, Lyles RH, et al:: Local recurrence after skin-sparing mastectomy: tumor biology or surgical conservatism? Ann Surg Oncol 2003, 10:108-112.

7. Simmons RM, Fish SK, Gayle L, et al.: Local and distant recurrence rates in skin-sparing mastectomy compared with nonskin-sparing mastectomies. Ann Surg Oncol 1999, 6:676-678.

8. Slavin SA, Schnitt SJ, Duda RB, et al.: Skin-sparing mastectomy and immediate reconstruction: oncologic risks and aesthetic results in patients with early stage breast cancer. Plast Reconstr Surg 1998, 102:49-62.

9. Kroll SS, Khoo A, Singletary SE, et al.: Local recurrence risk after skin-sparing and conventional mastectomy: a 6-year follow-up. Plast Reconstr Surg 1999, 104:421-425.

10. Medina-Franco H, Vasconez LO, Fix RJ, et al:: Factors associated with local recurrence after skin-sparing mastectomy and immediate breast reconstruction for invasive breast cancer. Ann Surg 2002, 235:814-819.

11. Beer GM, Varga Z, Budi S, et al:: Incidence of the superficial fascia and its relevance in skin-sparing mastectomy. Cancer 2002, 94:1619-1625. 
12. Wellisch DK, Schain WS, Noone RB, Little JW 3rd: The psychological contribution of nipple addition in breast reconstruction. Plast Reconstr Surg 1987, 80:699-704.

13. Cense HA, Rutgers EJ, Lopes-Cardozo M, Van Lanschot JJ: Nipple sparing mastectomy in breast cancer: a viable option? Eur J Surg Oncol 2001, 27:521-526.

14. Lambert PA, Kolm P, Perry RR: Parameters that predict nipple involvement in breast cancer. J Am Coll Surg 2000, 191:354359.

15. Gajdos C, Tartter PI, Bleiweiss IJ: Subareolar breast cancers. Am J Surg 2000, 180:167-170.

16. Vyas JJ, Chinoy RF, Vaidya JS: Prediction of nipple and areola involvement in breast cancer. Eur J Surg Oncol 1998, 24:1516.

17. Laronga C, Kemp B, Johnston D, Robb GL, Singletary SE: The incidence of occult nipple-areola complex involvement in breast cancer patients receiving a skin sparing mastectomy. Ann Surg Oncol 1999, 6:609-613.

18. Gerber B, Krause A, Reimer T, et al.: Skin-sparing mastectomy with conservation of the nipple-areola complex and autologous reconstruction is an oncologically safe procedure. Ann Surg 2003, 238:120-127.

19. Merrick HW, Battle JA, Padgett BJ, Dobelbower RR: ELIOT for early breast cancer: a report on long-term results. Front Radiat Ther Oncol 1997, 31:126-130.

20. Petit JY, Veronesi U, Orecchia R, et al.: The nipple-sparing mastectomy: early results of a feasibility study of a new application of perioperative radiotherapy (ELIOT) in the treatment of breast cancer when mastectomy is indicated. Tumori 2003, 89: 288-291.

21. DuBois J-B, Hay M, Gely S, Saint-Aubert B, Rouanet P, Pujol H: ELIOT in breast carcinomas. Front Radiat Ther Oncol 1997, 31: 131-137.

22. Battle JA, DuBois J-B, Merrick HW, Dobelbower RR: ELIOT for breast cancer. In Current Clinical Oncology: Intraoperative Irradiation: Techniques and Results. Edited by Gunderson LL, et al. New York: Humana Press, Inc.: 1999:521-526.

23. Veronesi $U$, Orecchia R, Luini $A$, et al.: Focalised intraoperative irradiation after conservative surgery for early stage breast cancer. Breast 2001, 10(Suppl 3):84-89.

24. Veronesi $U$, Orecchia $R$, Luini $A$, et al:: A preliminary report of intraoperative radiotherapy (ELIOT) in limited-stage breast cancers that are conservatively treated. Eur J Cancer 2001, 37:2178-2183.

25. Benediktsson KP, Perbeck L, Geigant E, Solders G: Touch sensibility in the breast after subcutaneous mastectomy and immediate reconstruction with a prosthesis. $\mathrm{Br} J$ Plast Surg 1997, 50:443-449.

26. Simmons RM, Brennan $M$, Christos $P$, et al.: Analysis of nipple/ areolar involvement with mastectomy: can areola be preserved? Ann Surg Oncol 2002, 9:165-168.

27. Chagpar $A B$ : Skin-sparing and nipple-sparing mastectomy: preoperative, intraoperative and postoperative considerations. Am Surg 2004, 70:425-432.

28. Crowe JP Jr, Kim JA, Yetman R, Bambury J, Patrick RJ, Baynes D: Nipple-sparing mastectomy: technique and results of 54 procedures. Arch Surg 2004, 139:148-150.

29. Simmons Rm, Hollenbeck ST, Latrenta GS: Areola-sparing mastectomy with immediate breast reconstruction. Ann Plast Surg 2003, 51:547-551.

\section{S11 \\ Partial breast irradiation and intraoperative radiotherapy \\ R Orecchia ${ }^{1,2}$}

${ }^{1}$ Department of Radio-Oncology, European Institute of Oncology, Milan, Italy; ${ }^{2}$ University of Milan School of Medicine, Milan, Italy

Breast Cancer Research 2007, 9(Suppl 1):S11 (doi: 10.1186/bcr1694)

Over the past few years an increasing number of papers have appeared in peer-reviewed journals detailing various partial breast irradiation (PBI) approaches, utilizing high-precision external beam radiation therapy, single-dose intraoperative radiation therapy or brachytherapy. The concept of PBI has been associated with the use of accelerated schedules of fractionation. More recently, several preliminary clinical reports of retrospective series and the 5-year results of the only randomized study from Budapest using brachytherapy have produced significant discussion and confirmed the widespread interest toward $\mathrm{PBI}$. The comparison between the current standard with early data coming from $\mathrm{PBI}$ techniques poses a dilemma as to when preliminary results are sufficiently mature to consider a new treatment approach as safe.

$\mathrm{PBI}$ allows reducing the radiation field to only the initially involved quadrant of the breast and significantly shortens the duration of radiation therapy. This may represent the possibility of overcoming constraints such as accessibility to the radiation therapy centres, and the socioeconomic impact on the working life and on the personal habits of the patient. PBI seems to have a positive impact on patients' quality of life. Another important advantage is the avoidance of interactions with systemic therapy that may determine delays in the initiation or in the carrying out of the conventional treatment.

These possible benefits must be balanced with the potential risk of recurrence within the untreated tissue in the breast receiving $\mathrm{PBI}$ as well as the unknown long-term cosmetic results with the accelerated techniques. Significant practical considerations also include the choice of technique, interstitial brachytherapy (high dose-rate versus low doserate), balloon-based brachytherapy (MammoSite ${ }^{\circledR}$ ), external beam (3Dconformal versus intensity modulated radiation therapy), and intraoperative irradiation (electrons versus low-energy X-ray device). The choice of the technique has an impact on the schedules and dose-rate that can be used, on the volume that can be irradiated and on the dose homogeneity achievable.

Patient selection also remains uncertain, including questions regarding age exclusions, applicability with various primary tumour sizes, hystopathological features, the tumour-free margin and amount of tumour near the margins, and negative (including micrometastasis) or positive axillary lymph node status (up to three or more).

For this reason, data coming from the multicentric or unicentric large phase III ongoing trials in the United States and Europe comparing standard irradiation with the different PBI schedules and techniques will hopefully support the movement of PBI into routine clinical practice. Issues of patient selection, target volume definition, total dose, fractionation, and the quality assurance programme should be addressed and defined from analysing the results of such randomized trials.

Acknowledgements The authors thank the support of the American Italian Cancer Foundation and Associazione Italiana Ricerca sul Cancro to the research programme of the division. 


\section{SYMPOSIUM III}

\section{Controversies in Clinical Practice (Part I)}

\section{S12}

\section{Current status of adjuvant endocrine therapy for premenopausal patients with primary breast cancer A Howell}

CRUK Department of Medical Oncology, University of Manchester, Christie Hospital NHS Foundation Trust, Manchester, UK

Breast Cancer Research 2007, 9(Suppl 1):S12 (doi: 10.1186/bcr1695)

The increased risk of death from cancer in young women attests to its aggressive nature and a greater likelihood of being oestrogen receptor (ER)-negative. Approximately $60 \%$ of tumours are ER-positive, however, and thus endocrine therapy is an important consideration in this group. In some countries chemotherapy alone was used for many years until the importance of the additional therapeutic effect of amenorrhea became evident and later, after much scepticism, tamoxifen was shown to be effective in this group of women. Sadly this rather staccato history of treatment has left many questions concerning endocrine therapy in young women unanswered. For example, there have been at least nine studies that show ovarian suppression is equivalent for disease-free and overall survival to chemotherapy, showing equivalent therapeutic advantage. However, the correct design of their studies should have included a third arm of chemotherapy and ovarian ablation and some randomisation to investigate the role of additional tamoxifen. New trials such as SOFT, TEXT and PROMISE will help answer the question of optimal endocrine therapy \pm chemotherapy in young women and will also help decide whether aromatase inhibitors are effective in the presence of ovarian suppression. Two further points are important to consider. One is that chemotherapy used alone in very young women with ER-positive disease is detrimental since they do not have chemotherapy-induced amenorrhoea. Secondly, the value of chemotherapy in strongly ER-positive disease is being increasingly questioned.

References

1. Brown RJ, Davidson NE: Adjuvant hormonal therapy for premenopausal women with breast cancer. Semin Oncol 2006, 33:657-663.

2. Colleoni M, Gelber S, Goldhirsch A, Aebi S, Castiglione-Gertsch M, Price KN, Coates AS, Gelber RSP: International Breast cancer Study Group: Tamoxifen after adjuvant chemotherapy for premenopausal women with lymph node positive breast cancer. J Clin Oncol 2006, 24:1332-1341.

3. Goldstein LJ: Controversies in adjuvant endocrine treatment of premenopausal women. Clin Breast Cancer 2006, 6:S36-S40.

4. Kaufmann M, Jonat W, Blamey R, Cuzick J, Namer M, Fogelman I, de Haes JC, Schumacher M, Sauerbrei W, Zoladex Early Breast Cancer Research Association (ZEBRA) Trialists Group: Survival analyses from the ZEBRA study. Goserelin (Zoladex) versus CMF in premenopausal women with node positive breast cancer. Eur J Cancer 2003, 39:1711-1717.

\section{S13}

Triple-negative (basal-like) breast cancer: a new entity LA Carey

Hematology/Oncology Department, University of North Carolina, Chapel Hill, NC, USA

Breast Cancer Research 2007, 9(Suppl 1):S13 (doi: 10.1186/bcr1696) Unsupervised gene expression array profiling has provided biological evidence for the heterogeneity of breast cancer through the identification of intrinsic subtypes such as luminal A, luminal B, HER2+/ER- and the basal-like subtype. The basal-like subtype is characterized by low expression of both the ER and HER2 clusters of genes, so is typically ER-negative, PR-negative, and HER2-negative on clinical testing; for this reason, it is often referred to as 'triple-negative' breast cancer.
Basal-like breast cancer has some unique characteristics. It is the type of breast cancer that BRCA1 mutation carriers generally develop, although most basal-like breast cancers are sporadic. It comprises approximately $15 \%$ of all breast cancers, but is overrepresented among young AfricanAmerican women who develop breast cancer, in whom it comprises 39\%. Although it does not occur at higher stages than other breast cancer subtypes, it is usually high grade and highly proliferative, which may explain the poor prognosis associated with this subtype in several series. Given the triple-negative status of basal-like breast cancer, it cannot be treated with ER-targeted or HER2-targeted therapies, so is primarily treated with chemotherapy. Fortunately, advances in adjuvant therapy appear to benefit ER-negative breast cancer even more than ERpositive breast cancer, and women with the basal-like subtype have similarly benefited. In addition, in cohorts of breast cancer treated with neoadjuvant chemotherapy, the pathologic complete response to anthracycline/taxane-based therapy was significantly higher among basal-like breast cancers than luminal breast cancers. The women with pathologic complete response have good outcome; the poor prognosis of basal-like breast cancer appears to relate to a particularly high risk of early distant relapse among those that had residual disease. Among the targeted agents for breast cancer, bevacizumab added to paclitaxel in a randomized phase III trial appeared to have similar benefit in the triple-negative subset as the other breast cancer phenotypes, suggesting effectiveness in basal-like breast cancer. Current investigations into other therapeutic options for this subtype include confirmation of effectiveness of VEGF targeting, examination of EGFR-targeted strategies, determining whether the association of basal-like breast cancer with BRCA1 mutations means that this DNA repair pathway is dysfunctional in all basal-like breast cancer, and determining whether specific chemotherapy agents have greater or lesser efficacy in this subtype. Future studies should also focus upon identifying whether there are particular genetic or environmental risk factors for basal-like breast cancer that differ from other subtypes, and whether there are metastatic or predictive signatures that can be identified within the basal-like subtype.

\section{S14}

Treatment of metastatic disease after current adjuvant approaches (taxanes, aromatase inhibitors, trastuzumab)

\section{PF Conte, V Guarneri, C Bengala}

Division of Medical Oncology, Department of Oncology and Hematology, University Hospital, University of Modena and Reggio Emilia, Modena, Italy

Breast Cancer Research 2007, 9(Suppl 1):S14 (doi: 10.1186/bcr1697)

Metastatic breast cancer patients represent a very heterogeneous population and several factors are important for therapeutic decision: patient characteristics including age, comorbidities, and performance status, tumor biological characteristics, site and extension of metastatic spread, prior adjuvant therapies and disease-free interval. In the past years, adjuvant treatment has been rapidly changing as new cytotoxics, new endocrine agents and targeted therapies are becoming the mainstay of treatment [1]. For endocrine-sensitive breast cancer, several large randomized trials have shown that third-generation aromatase inhibitors, can reduce the risk of relapse of early breast cancer in comparison with tamoxifen. Nowadays, aromatase inhibitors administered up front, as a switch after 2-3 years of tamoxifen or as extended treatment after 5 years of tamoxifen represent the standard treatment in postmenopausal women. As anthracycline and taxanes are the most active cytotoxic agents in breast cancer, anthracycline/taxanecontaining regimens are becoming the mainstay of adjuvant chemotherapy. Finally, the growing understanding of the biology of breast cancer cells led to identification of key molecular points that represent possible therapeutic targets. Trastuzumab, the monoclonal antibody against the HER-2 receptor, is approved for the treatment of high-risk early breast cancer overexpressing HER-2 and represents the standard treatment in these patients. With increasing use of very active drugs in the adjuvant setting, there is a greater need for effective 
therapy at the time of relapse [2]. In endocrine-sensitive breast cancer, sequencing data for anastrozole and tamoxifen have shown that tamoxifen is likely to be effective after progression on anastrozole. Moreover, in recent years Fulvestran, a selective ER downregulator acting as a pure antiestrogen agent, has been developed. Tamoxifen, Fulvestran or an aromatase inactivator are possible options after failure of adjuvant aromatase inhibitors. At present, it is not possible to define the optimal sequence of these endocrine agents. In endocrine-resistant disease, a treatment-free interval (TFI) $>12$ months after adjuvant chemotherapy has been shown to be an important factor for determining sensitivity to drug rechallenge. If the tumor has been exposed to an anthracycline and a taxane in adjuvant setting and the TFI is $>12$ months, the re-treatment with the same agents may be an option [3]. If the TFI is $<12$ months it is preferable to use a different agent. Capecitabine, gemcitabine and vinorelbine have demonstrated substantial activity in metastatic breast cancer. Finally, patients with HER2-positive tumors receiving adjuvant trastuzumab might be refractory (primary resistance) or might become resistant (secondary resistance) to trastuzumab. The mechanisms of primary and secondary trastuzumab resistance are not yet fully elucidated. Lapatinib, a new target agent that simultaneously inhibits both HER-2 and EGFR tyrosine kinases, has been shown to be active in trastuzumab-resistant metastatic breast cancer. Moreover, several new agents targeting HER-2 are currently under clinical development. There are no data on rechallenge with trastuzumab in patients who had received this agent as adjuvant treatment and relapsed after a long TFI, and this issue is a new area of research.

References

1. Conte PF, Bengala C, Guarneri V: Controversies of chemotherapy for the treatment of metastatic breast cancer. Eur J Cancer 2007, Suppl 5:11-16.

2. Conte PF, Guarneri V: The curability of breast cancer and the treatment of advanced disease. Eur J Nucl Med Mol Imaging 2004, 31(Suppl 1):S149-S161.

3. Gennari A, Bruzzi P, Orlandini C, et al.: Activity of first line epirubicin and paclitaxel in metastatic breast cancer is independent of type of adjuvant therapy. $\mathrm{Br} J$ Cancer 2004, 90: 962-967.

\section{S15}

\section{Present and future role of bisphosphonates in treatment GN Hortobagyi}

Department of Breast Medical Oncology, The University of Texas MD Anderson Cancer Center, Houston, TX, USA

Breast Cancer Research 2007, 9(Suppl 1):S15 (doi: 10.1186/bcr1698) Bone metastases are part of a spectrum of conditions based on dysregulated osseous metabolism. Metastatic cancer cells activate osteoclasts, both directly, by producing and releasing local humoral factors that result in osteoclast activation, and by activating osteoblasts, which in turn further affect osteoclast activity. Activated osteoclasts, when uncoupled and unbalanced from osteoblast activity, result in net excess bone resorption, resulting in bone loss and eventually lytic or mixed metastatic lesions. Therefore, the osteoclast, and the humoral activation system that leads to osteoclast recruitment and activation have become the major targets for developing therapies active in bone metastases. Bisphosphonates are potent osteoclast inhibitors and interfere with recruitment of osteoclast precursors, activation and release of bone resorbing substances. Bisphosphonates represent the treatment of choice for osteoporosis. They might also have weak, but direct, antitumor effects. As single agents, bisphosphonates relieve pain, and reduce the skeletal complication rate associated with bone metastases. Prospective randomized trials have clearly demonstrated that when added to standard antitumor therapy, bisphosphonates reduce skeletal morbidity, fractures, pain and analgesic requirements, as well as the need for radiotherapy or orthopedic interventions. Bisphosphonates also increase the time to first skeletal event, thus metastases. These observations have been made in a variety of tumor types. The optimal duration of bisphosphonate therapy has not been determined, and while the usual schedule of administration is monthly intravenous therapy for this indication, there are ongoing trials attempting to determine the most effective and safest schedule for patients with bone metastases. Similar studies are ongoing for patients with osteoporosis and osteopenia. Although bisphosphonates are very well tolerated, the last few years have brought a number of anecdotal reports of osteonecrosis of the jaw associated with bisphosphonate administration. More recently, systematic retrospective analyses of large databases have indicated a low but definite incidence of this complication, predominantly in patients receiving third-generation, potent bisphosphonates. These reports suggest the incidence to be in the range of $0.1-2.0 \%$, varying with tumor type and research methodology. There is insufficient information about prevention and management of this condition, although risk factors include poor oral hygiene, oral/dental interventions, increasing cumulative dose of bisphosphonates and duration of therapy.

There is much interest and ongoing trials to define the role of bisphosphonates in the preventive treatment of primary breast cancer. Whether bisphosphonates can prevent osseous metastases or not, emerging information about their use in treatment-induced bone loss might lead to the incorporation of these agents into the combined modality management of primary breast cancer anyway. This makes the determination of therapeutic benefit, optimal dose and schedule of administration very important.

\section{SYMPOSIUM IV}

Primary Systemic Therapy and Adjuvant Treatment

\section{S16}

\section{Standards and future strategies in adjuvant chemotherapy treatments C Hudis, H McArthur}

Memorial Sloan-Kettering Cancer Center, New York, NY, USA

Breast Cancer Research 2007, 9(Suppl 1):S16 (doi: 10.1186/bcr1699)

Breast cancer is an increasing global public health burden with more than 1 million new cases anticipated worldwide and more than 200,000 new cases anticipated in the United States in 2007. Screening and awareness have increased the detection of breast cancer at its early stages where it is most curable, but many such patients have microscopic metastatic disease even when diagnosed. The outcome for such patients can only be improved through the effective control of this undetected systemic disease, thus motivating adjuvant therapy. For patients with sufficient risk, based historically on nodal status, tumor size, specific histology, and hormone receptor status, chemotherapy can be the only option or a component of regimens including hormonal agents and anti-HER2 drugs. Genetic profiling is a recent addition to our risk stratification methods and can allow for more precise selection of chemotherapy. Historical chemotherapy regimens were largely empiric combinations of drugs with proven activity in advanced disease. More recent advances have been translational, with preclinical development of the taxanes leading to their testing in the adjuvant setting and mathematical modeling leading to improvements in the delivery of otherwise standard agents. Despite this mixture of empiricism and translational research, adjuvant chemotherapy is highly effective, particularly in selected subsets of patients, such as those with no expression of hormone receptors. Building on the demonstration of activity for the humanized monoclonal anti-HER2 antibody (trastuzumab) as adjuvant treatment, newer HER2 targeting drugs are in development and will be tested in this setting (lapatinib). For the majority of tumors lacking overexpression of HER2, planned research focuses on anti-angiogenic agents (bevacizumab). For many patients, an optimized chemotherapy plan (that is, dose- 
dense administration of anthracyclines and taxanes) combined with appropriate targeted agents is likely to offer even larger improvements in outcomes than those already achieved.

\section{S17}

\section{Adjuvant hormonal therapy for postmenopausal women \\ EP Winer}

Dana-Farber Cancer Institute and Harvard Medical School, Boston, MA, USA

Breast Cancer Research 2007, 9(Suppl 1):S17 (doi: 10.1186/bcr1700)

Approximately $75 \%$ of all breast cancer is hormone receptor positive and the majority arises in postmenopausal women. In the United States alone, there are over 100,000 women annually who are diagnosed with postmenopausal hormone-receptor-positive disease. Adjuvant hormonal therapy substantially decreases a woman's risk of developing recurrent breast cancer and improves overall mortality. For almost two decades, a 5-year course of tamoxifen was the standard treatment offered to almost all women with hormone-receptor-positive disease. Over the past decade, the aromatase inhibitors have emerged as highly effective agents in the treatment of hormone-receptor-positive breast cancer. Multiple large trials including 25,000 women have assessed the role of the aromatase inhibitors in the adjuvant setting. These studies have demonstrated that the use of an aromatase inhibitor, either in place of tamoxifen or following a 2-year to 5-year course of tamoxifen, will reduce the risk of disease recurrence compared with the use of a 5 -year course of tamoxifen alone. To date, these studies have not demonstrated a substantial improvement in overall survival, but the follow-up was relatively short. The optimal treatment approach has not been identified. Recently completed and ongoing trials are comparing a 5 -year course of an aromatase inhibitor with the use of sequential therapy. Given the natural history of hormone-receptor-positive breast cancer, there is also great interest in treatment strategies that extend beyond 5 years. The toxicity profile of the aromatase inhibitors differs from tamoxifen, and these differences need to be considered in making clinical decisions. Ultimately, it is probable that different treatment approaches will be appropriate for different women based on tumor biology, pharmacogenetic variability, and susceptibility to side effects.

\section{S18}

\section{Preoperative systemic treatment for operable disease: biological correlates of response to neoadjuvant treatment GN Hortobagyi}

Department of Breast Medical Oncology, The University of Texas MD Anderson Cancer Center, Houston, TX, USA

Breast Cancer Research 2007, 9(Suppl 1):S18 (doi: 10.1186/bcr1701) Experiments on preclinical models have suggested that preoperative introduction of chemotherapy of endocrine therapy can improve survival by abrogating a postsurgical growth spurt and by addressing micrometastatic disease at an earlier stage than when given postoperatively. Clinical trials have not confirmed this hypothesis, although they have demonstrated that preoperative systemic therapy reduced the overall tumor burden, expanded the indications for breastconserving surgery to patients with more advanced disease, and provided an in vivo assessment of response that facilitated the safe and effective administration of systemic treatment. Preoperative administration of systemic therapy also provides an improved investigational model, since sequential monitoring with the primary tumor in situ offers the opportunity for multiple biopsies to monitor the biological effects of treatment. Large randomized trials have shown preoperative chemotherapy to be at least equivalent in disease-free and overall survival to the same chemotherapy administered postoperatively. Emerging data suggest, however, that the effects of systemic therapy in general, and preoperative therapy in particular, vary with different subclasses of breast cancer (BC). The first such observations were based on the estrogen receptor (ER). It is now widely accepted that the magnitude of benefit from chemotherapy is proportionately more modest for patients with ER+ tumors than for ER- tumors. This is dramatically expressed in preoperative trials, where the pathological complete remission ( $\mathrm{pCR}$ ) rate is fourfold to sixfold higher for ER- BC than for ER+ BC. Similar variation in the pCR rate is observed by grade. More recent reports have indicated that the $\mathrm{pCR}$ rate was very low for invasive lobular cancers (almost always ER+) compared with invasive ductal cancers. Paradoxically, although pCR identifies a group of patients with improved survival compared with patients who do not achieve $\mathrm{pCR}$, patient groups with lower $\mathrm{PCR}$ rates (those with ER+ tumors, those with low grade or lobular cancer) have better overall survival than those who tend to have higher pCR rates. This observation emphasizes the importance of understanding the biological heterogeneity of $\mathrm{BC}$, and parallels the observations made in lymphoma over the past several decades: high-grade lymphomas respond more readily to chemotherapy but have lower survival rates in early follow-up than low-grade lymphomas. Studies based on gene expression profiling in $\mathrm{BC}$ have confirmed the existence of at least three distinct forms of BC: ER+/HER2-, HER2+, and 'triple-negative'. These three groups differ by much more than the individual gene (ER or HER2) expression, and their clinical course and responsiveness to different treatments is quite different too. Thus, HER2+ and triplenegative tumors achieve a high pCR rate $(40-50 \%)$ with standard combinations, while ER+/HER2- tumors do not ( $p$ CR rate $<10 \%$ ). There are other ramifications to drug sensitivity, and, most importantly, gene profiling leads to the identification of potential new targets for therapeutic intervention. Validation of such novel targets and development of specific therapeutics might be the best legacy of neoadjuvant treatment.

\section{S19}

\section{Neoadjuvant endocrine therapy: clinical and biological issues \\ IE Smith}

The Royal Marsden Hospital and Institute of Cancer Research, London, UK

Breast Cancer Research 2007, 9(Suppl 1):S19 (doi: 10.1186/bcr1702)

Background Neoadjuvant endocrine therapy is being increasingly used with two aims: to downstage cancers and reduce the need for mastectomy; and to establish short-term clinical and/or biological surrogate endpoints predicting for long-term outcome.

Methods The results of key randomised trials addressing these issues are reviewed.

Results In the P24 trial for patients not suitable for conservative surgery, neoadjuvant letrozole was shown to achieve a significantly greater clinical overall response rate than tamoxifen (56\% versus $36 \%$, $P<0.001)$ and a significantly better incidence of breast-conserving surgery ( $45 \%$ versus $35 \%, P=0.02$ ). In the IMPACT trial, neoadjuvant anastrozole was compared with tamoxifen and the combination (three arms, equivalent to adjuvant ATAC). No significant difference in clinical overall response was seen between the three arms although breastconserving surgery was considered feasibly significantly more often with anastrozole $(46 \%)$ than with tamoxifen $(22 \%)$ or with the combination $(26 \%)(P=0.03)$. In the PROACT trial, neoadjuvant anastrozole achieved a higher clinical overall response rate than tamoxifen (50\% versus $40 \%$ ) but this difference was not significant (in the endocrine therapy alone group - excluding patients also treated with chemotherapy). Breast-conserving surgery was achieved significantly more often with anastrozole $(43 \%$ versus $31 \%, P=0.04)$.

The optimum duration of neoadjuvant endocrine therapy prior to surgery has not been established but circumstantial evidence suggests that it is at least 4 months, and probably longer.

In the P24 trial and the IMPACT trial, patients with higher levels of ER expression had a significantly higher chance of clinical response. Likewise in both trials, aromatase inhibitors appeared strikingly more effective than tamoxifen in the subgroup of patients with HER2positive, ER-positive tumours. 
In the IMPACT trial, the change in proliferation rates after 2 weeks of treatment as measured by Ki67 was significantly greater for anastrozole than for tamoxifen or the combination, reflecting results in the adjuvant ATAC trial, and suggesting that Ki67 might be a better marker for long-term outcome than clinical response. Likewise, longterm follow-up of the IMPACT trial showed that the absolute level of Ki67 suppression after 2 weeks of neoadjuvant endocrine therapy significantly correlated with long-term disease-free survival outcome, and more so than baseline pre-treatment Ki67. Changes in expression of $>2,800$ genes were observed after 2 weeks of aromatase inhibitor therapy.

Conclusion These results suggest that neoadjuvant endocrine therapy is an effective clinical strategy to reduce the need for mastectomy in postmenopausal women with large hormone receptor-positive breast cancer. Short-term changes in molecular markers may be superior to clinical response in predicting long-term outcome, and this hypothesis is being further tested in a large prospective randomised trial.

\section{SYMPOSIUM V}

\section{Advances in Metastatic Disease}

\section{$\mathbf{S 2 0}$}

\section{Chemotherapy in metastatic disease: an overview} IE Smith

The Royal Marsden Hospital and Institute of Cancer Research, London, UK

Breast Cancer Research 2007, 9(Suppl 1):S20 (doi: 10.1186/bcr1703)

Many different cytotoxic agents are currently available for the treatment of metastatic breast cancer (MBC), and multiple factors determine the choice of treatment. These include previous adjuvant therapy, tumour characteristics (for example, molecular receptors, sites of relapse), patient characteristics (for example, performance status, age) and patient preference.

The aims of treatment with chemotherapy for MBC are: to prolong survival; to relieve symptoms; to improve and maintain quality of life; and to delay disease progression.

Only a small minority of MBC chemotherapy trials has shown a significant survival benefit, and to some extent this is explained by the multiple lines of potential further therapy following completion of the trial. Survival is therefore an uncertain endpoint in MBC trials.

Symptom improvement has been shown to correlate with response rate in one trial but this important endpoint is not used often enough. Quality-of-life endpoints are appropriate in principle, but in reality many trials have shown a poor correlation with outcome, suggesting that this tool may be relatively insensitive in this context. Time to disease progression is a much used and important endpoint because it takes into account the fact that symptom relief and prolonged survival relate not just to patients achieving objective response, but also to those with minor response or stable disease.

Specific issues relating to chemotherapy for MBC include the following. There is evidence from older trials supporting the principle that endocrine therapy should be used before chemotherapy in patients with nonlife-threatening hormone-receptor-positive disease. Patients whose primary is HER2-unknown should have this checked at the time of relapse to determine whether trastuzumab should be given in addition to chemotherapy. Ideally, patients with primary HER2negative disease should have metastatic disease rechecked since there is evidence of HER2-positive conversion in up to $25 \%$ of such patients. The choice of combination versus single-agent sequential chemotherapy is a complex one. The weight of current evidence suggests no significant survival difference with the combination, and this may be associated with more toxicity. There is no absolute answer here; choice depends on the nature of the drugs being used.
Multiple lines of chemotherapy are now available for MBC. Current evidence suggests that it is only worth proceeding to third-line treatment if there has been a response to first-line and/or second-line therapy. New drug trials are appropriate for fit patients who have failed two lines of conventional therapy, and sometimes earlier than this in patients with nonlife-threatening disease.

\section{SYMPOSIUM VI}

\section{Targeted Therapy: Present and Future}

\section{S21}

Epigenetics of breast cancer M Esteller

Cancer Epigenetics Laboratory, Spanish National Cancer Centre (CNIO), Madrid, Spain

Breast Cancer Research 2007, 9(Suppl 1):S21 (doi: 10.1186/bcr1704)

In breast cancer there is a global $40 \%$ loss of methylated cytosine, but DNA hypermethylation indicates which genes are turned off in tumors and tumor cell lines. Every tumor type has its own pattern of hypermethylation. Genes important in familial breast cancer are also epigenetically silenced. In sporatic tumors, BRCA1 expression was wiped out by a combination of loss of heterozygosity (gene deletion) and epigenetic silencing (hypermethylation). In familial BRCA1 tumors, hypermethylation occasionally serves as an alternate mechanism for the 'second hit', when there was not loss of heterozygosity. Other known cancer genes are hypermethylated in breast cancers, such as those that affect cell cycle (p16 INK4a), and hormone receptors (ER, PR). New epigenomic approaches revealed the novel importance of potential tumor suppressor genes, such as the prolactin receptor and WRN, the Werner syndrome gene associated with premature aging and increased cancer risk, which is silenced in a subset of breast cancers. In addition to direct DNA hypermethylation, modification of histones is another epigenetic mechanism with implications in breast cancer. Overall, there is global loss of monomethylation and trimethylation of histone $\mathrm{H} 4$ in cancer. New epigenetic drugs targeting DNA methylation and histone deacetylation are in development for the treatment of breast cancer.

\section{S22}

\section{Present and future roles of bevacizumab in breast cancer}

\section{H Cortés-Funes}

Hospital Universitario 12 de Octubre, Madrid, Spain

Breast Cancer Research 2007, 9(Suppl 1):S22 (doi: 10.1186/bcr1705)

Vascular endothelial growth factor (VEGF), a potent angiogenic factor, has been reported to be associated with a poor prognosis in primary breast cancer and other solid tumours [1]. VEGF was observed to be one of the most important mediators of tumour angiogenesis in human breast cancer and was the only one linked to poor relapse-free survival [2].

VEGF affects tumour growth and metastasis in many forms of breast cancer, including invasive/noninvasive, node-negative/node-positive, inflammatory and metastatic disease. Also, VEGF expression has been observed across a number of disease-related variables, including hormonal and HER-2 status.

A complex relationship between oestrogen, the oestrogen-receptor subtypes, and VEGF has been seen in preclinical breast cancer models. Oestrogen modulates VEGF expression at the gene transcriptional level [3]. VEGF expression in breast cancer cells promotes oestrogen-independent tumour growth in ovariectomized mice and VEGF-stimulated neovascularization, and contributed to tumour growth in both oestrogen-treated and nonoestrogen-treated mice. Tumour growth was highest in VEGF-expressing mice treated with oestrogen [4]. 
In multiple prospective and retrospective studies assessing blood and tumour samples in lung cancer patients, VEGF and angiogenesis have been associated with larger tumour size, increased rate of metastasis, recurrence of disease and poorer overall and disease-free survival [5-7]. Bevacizumab is a humanized recombinant antibody that prevents VEGF receptor binding, and inhibits angiogenesis and tumour growth. In patients receiving an irinotecan plus fluorouracil/leucovorin regimen for first-line treatment of metastatic colorectal cancer, the addition of bevacizumab significantly increased overall survival. In the second-line treatment of advanced colorectal cancer, patients who received bevacizumab in combination with a fluorouracil/leucovorin plus oxaliplatin (FOLFOX4) regimen had an overall survival time 2 months longer than that in patients receiving FOLFOX4.

Bevacizumab has been tested in many tumours, proving efficacy when added to almost any chemotherapy regimen. It has been hypothesized that there are three main mechanisms by which bevacizumab exerts its antitumour activity: regression of existing tumour microvasculature, normalization of surviving mature tumour vasculature and inhibition of vessel regrowth and neovascularization as an effect with continuous treatment [8].

Based on preclinical findings that have shown the activity of bevacizumab in breast cancer, bevacizumab monotherapy was tested in a phase $1 / 1$ ll dose escalation trial (AVF0776g) in metastatic breast cancer (MBC) with three doses (3, 10 and $20 \mathrm{mg} / \mathrm{kg}$ every 2 weeks) in 75 patients who had relapsed following at least one conventional chemotherapy regimen for metastatic disease. Combining data from the three arms, the overall response (OR) rate was $6.7 \%$, the median duration of response (DR) was 5.6 months and overall survival (OS) was 10.2 months. Bevacizumab was well tolerated although four patients presented with headache with nausea and vomiting at the higher dose (dose limiting toxicity). Comparison of data from the three study arms indicated that a bevacizumab dose of $10 \mathrm{mg} / \mathrm{kg}$ every 2 weeks produced the best therapeutic ratio in this population of heavily pretreated patients [9].

Bevacizumab has since been studied in several phase II trials with different chemotherapy regimens (docetaxel, vinorelbine), targeted agents (trastuzumab, erlotinib) and hormonal therapies (letrozol), showing encouraging results and acceptable toxicity profiles in these combinations. Nowadays, there is evidence for efficacy of bevacizumab from two phase III trials (AVF2119g and E2100). The first phase III trial published (AVF2119g) compared a combination of bevacizumab $\left(15 \mathrm{mg} / \mathrm{kg}\right.$ every 3 weeks plus capecitabine $2,500 \mathrm{mg} / \mathrm{m}^{2}$ daily for 2 weeks of a 3-week cycle) versus capecitabine alone. A total of 462 women with MBC previously treated with an anthracycline and a taxane were randomized to any of the study arms. The primary study endpoint was progression-free survival (PFS) and secondary endpoints included the OR rate, DR and OS. There was no significant difference in PFS between the two arms. The proportion of progression-free patients at 6 months was 33.8\% for the Xeloda-alone arm and 33.0\% for the Xeloda plus bevacizumab arm. Median PFS was 4.17 and 4.86 months, respectively $(\mathrm{HR}=0.98, P=0.857)$. Overall survival data for the Xeloda-alone arm (14.5 months) compared with the Xeloda plus bevacizumab arm (15.1 months) was also not significant. Although the addition of bevacizumab to capecitabine did improve PFS nor OS, there was a significant increase in the OR rate in the capecitabine plus bevacizumab arm compared with the capecitabine-only arm when the data were analysed both by investigators $(30.2 \%$ versus $19.1 \%$; $P=0.006)$ and by an independent review facility (19.8\% versus $9.1 \%$; $P=0.001)$. However, no significant differences in PFS or in OS were found between the two arms. The combination was found to be well tolerated, with no increase in the frequency or severity of capecitabinerelated adverse events in the bevacizumab-containing arm [10]. In this study, although the addition of bevacizumab to capecitabine did not improve PFS (the primary endpoint), the OR rate doubled.

There have been a number of reasons hypothesized that could explain these observations. First of all, it has been demonstrated that VEGF inhibition is more effective in earlier disease, since in advanced disease there are also other angiogenic factors that are overexpressed [11].
Another reason could be that patients were heavily pretreated, having received both an anthracycline and a taxane prior to entering the trial. The other phase III trial (E2100) is an ongoing open-label, phase III trial in which bevacizumab is being evaluated in combination with weekly paclitaxel as first-line therapy for MBC. Patients recruited to this trial are less heavily pretreated than patients in AVF2119g. A total of 722 patients have been randomized to one of two arms: paclitaxel $90 \mathrm{mg} / \mathrm{m}^{2}$ alone every week for 3 weeks followed by 1 week without treatment or paclitaxel plus bevacizumab $10 \mathrm{mg} / \mathrm{kg}$ every 2 weeks. No crossover is permitted in this trial.

The objectives are to compare PFS, the OR rate and OS between the arms. Preliminary results from the first preplanned interim analysis showed a significant increase in median PFS in patients receiving bevacizumab plus paclitaxel compared with paclitaxel alone (11.4 versus 6.11 months; $P<0.0001)$. This PFS result was obtained following $89 \%$ of the required events. The OR rate for all patients was $29.9 \%$ (bevacizumab/paclitaxel) versus $13.8 \%$ (paclitaxel) $(P<0.0001)$, and was $37.7 \%$ (bevacizumab/paclitaxel) versus $16 \%$ (paclitaxel) $(P<0.0001)$ for patients with measurable disease only. The survival data are still immature [12].

Differences in the patient populations may help to explain the differences in results between the two phase III trials. Trial AVF2119 included a higher proportion of patients who had received chemotherapy, including those who had received both an anthracycline and a taxane. A greater number of HER2-positive patients were also enrolled in AVF2119, most of whom had previously received trastuzumab.

Trial AVF2119 patients were generally more heavily pretreated than those enrolled in the E2100 trial. This may partly explain why the significant response rate improvement seen in this trial did not translate into a PFS benefit. Showing these results, it can be hypothesized that bevacizumab should be used as early as possible to obtain the maximum benefit.

There are many other ongoing phase III trials that are testing the use of bevacizumab in combination with standard chemotherapy regimens.

The AVADO trial is an ongoing randomized, double-blind, placebocontrolled, multicentre phase III trial that will randomize 705 patients to receive docetaxel at a dose of $100 \mathrm{mg} / \mathrm{m}^{2}$ every 3 weeks with either placebo, bevacizumab $7.5 \mathrm{mg} / \mathrm{kg}$ or bevacizumab $15 \mathrm{mg} / \mathrm{kg}$. The primary trial endpoint will be PFS. Secondary endpoints will include the OR rate, duration of response, time to treatment failure, overall survival, safety and quality of life. Another trial exploring bevacizumab in combination with standard regimens is the RIBBON-1 study, a phase III trial to be conducted in countries worldwide that will investigate further combinations of bevacizumab and chemotherapy. The primary trial endpoint is PFS. Investigators will assign patients to a chemotherapy regimen, including specified regimens of anthracycline-based combination chemotherapy, taxane (docetaxel or Abraxane ${ }^{\mathrm{TM}}$ ) every 3 weeks, or Xeloda. Patients are then randomized to receive bevacizumab $(15 \mathrm{mg} / \mathrm{kg}$ every 2 weeks) or placebo. Another combination that is also being explored is bevacizumab plus trastuzumab. There is a strong rational for combining both antibodies as the different factors that bevacizumab and trastuzumab inhibit play important roles in tumour growth and progression. It has been shown that activation of the HER family of receptors induces VEGF expression and angiogenesis in cancer cell models [13]. These observations suggest that combined inhibition of VEGF and HER2 may enhance antitumour activity.

The feasibility of combining bevacizumab with trastuzumab was evaluated in the phase I, open-label AVF2473s trial. A total of nine patients with HER2-positive advanced or MBC were treated in one of three cohorts. Data from this trial led to the recommendation of bevacizumab $10 \mathrm{mg} / \mathrm{kg}$ every 2 weeks for use in combination with trastuzumab $2 \mathrm{mg} / \mathrm{kg}$ weekly (after a loading dose of $4 \mathrm{mg} / \mathrm{kg}$ ) in phase II trials. The maximum tolerated dose was not reached. Analyses of pharmacokinetic parameters showed that coadministration of bevacizumab and trastuzumab does not alter the pharmacokinetics of either agent, indicating no interaction between the two agents. The combination was well tolerated, with no grade $3 / 4$ adverse events [14]. Recently presented at the San Antonio Breast Cancer Symposium 2006 have been the first efficacy data from an ongoing, 
nonrandomized, open-label, uncontrolled, phase II study trial (TORI-B-03) that explores the combination of bevacizumab plus trastuzumab for the first-line treatment of Her2-positive locally recurrent, surgically unresectable, or metastatic breast cancer. The interim data of 37 patients showed that the combination of both targeted agents induces an overall response rate of 54\% with an acceptable safety profile without inducing the typical chemotherapyrelated side effects.

There is currently running a phase III trial of bevacizumab, docetaxel and trastuzumab (AVEREL) in patients with previously untreated, HER2-positive MBC: 320 patients will be randomized to receive either docetaxel and trastuzumab or docetaxel, trastuzumab and bevacizumab until disease progression. Patients from the control arm will not be permitted to crossover to receive bevacizumab upon progression. The primary trial endpoint is PFS. Other endpoints include response rate, duration of response, OS and safety.

There is also evidence from phase II trials proving the efficacy of adding bevacizumab to other agents such as vinorelbine, erlotinib or letrozol. All this combinations have demonstrated encouraging results with acceptable toxicity profiles that warrant further exploration.

Besides the use of bevacizumab in the metastatic setting, it has been hypothesized that the greater benefit could be obtained when used in earlier stages. The progression of breast cancer is accompanied by the production of a wide array of proangiogenic growth factors that promote and support tumour growth. When tumours are small they secrete VEGF, which acts as a paracrine factor to induce endothelial cell proliferation and blood vessel formation, mediating tumour progression. As the tumour develops further, additional factors are also secreted [15]. This evidence suggests that the inhibition of VEGF may be more beneficial in earlier stages, as neoadjuvant or adjuvant treatment. There are many trials, planned and ongoing, exploring bevacizumab in the neoadjuvant setting. An ongoing, phase II trial of bevacizumab in combination with docetaxel in the neoadjuvant setting (AVF2307s) is being conducted in patients with locally unresectable breast cancer with or without metastasis. Patients receive bevacizumab $10 \mathrm{mg} / \mathrm{kg}$ every 2 weeks and docetaxel $35 \mathrm{mg} / \mathrm{m}^{2}$ weekly for the first 6 weeks of an 8-week cycle. After two cycles, patients with stable disease or response, undergo surgery and radiotherapy, followed by further chemotherapy (not containing bevacizumab). The study objectives are to evaluate the efficacy and safety of neoadjuvant bevacizumab with docetaxel in breast cancer patients. Preliminary efficacy data showed that bevacizumab plus docetaxel reduces tumour vascular permeability and microvessel density [16]. Combined response data for both study arms revealed seven complete responses (14.3\%), 32 partial responses $(65.3 \%)$, five patients with stable disease and a further five with progressive disease. This gave an OR rate of $79.6 \%$, with no difference between the study arms. Both PFS and OS were 40 months in the docetaxel arm and neither had been reached in the docetaxel plus bevacizumab arm [17]. TORI-B-02 is a phase II neoadjuvant trial currently being conducted at the University of California, Los Angeles. A total of 90 patients are being enrolled into four different treatment arms, in order to compare two different bevacizumab doses with placebo: Arm 1: bevacizumab $7.5 \mathrm{mg} / \mathrm{kg}$, followed by six cycles of bevacizumab $7.5 \mathrm{mg} / \mathrm{kg}$ in combination with TAC (docetaxel, doxorubicin and cyclophosphamide) every 3 weeks; Arm 2: placebo $7.5 \mathrm{mg} / \mathrm{kg}$, followed by six cycles of placebo $7.5 \mathrm{mg} / \mathrm{kg}$ in combination with TAC every 3 weeks; Arm 3: bevacizumab $15 \mathrm{mg} / \mathrm{kg}$, followed by six cycles of bevacizumab $15 \mathrm{mg} / \mathrm{kg}$ in combination with TAC every 3 weeks; and Arm 4: placebo $15 \mathrm{mg} / \mathrm{kg}$, followed by six cycles of placebo $15 \mathrm{mg} / \mathrm{kg}$ in combination with TAC every 3 weeks. Then, 28-42 days after receiving chemotherapy, patients eligible for surgery will undergo resection. After either receiving surgery or completing chemotherapy, patients in Arms 1 and 3 will receive bevacizumab at their previous dose until disease progression. Patients in Arms 2 and 4 will not receive further therapy before disease progression. The primary objectives of the TORI-B-02 trial are to evaluate the safety and toxicity of bevacizumab given as preoperative therapy to patients with stage II/III breast cancer in combination with the TAC in order to compare tumour angiogenesis in patients given bevacizumab and placebo.

Other trial objectives include investigation of the clinical benefit of adding bevacizumab to TAC in the neoadjuvant setting. Parameters to be evaluated are the clinical objective response rate (complete response plus partial response), pathologic complete response rate and rate of breast-conserving surgery. As wound-healing complications have previously been reported in bevacizumab clinical trials, the effect of bevacizumab on postsurgical wound-healing will also be investigated as well as the rate of cardiac heart failure.

The most successful use of antiangiogenic therapy has been predicted to be in the adjuvant treatment, and there is a clear biologic basis and rationale for exploring bevacizumab in this clinical setting. A large proportion of patients with breast cancer have been reported to have primary tumours that overexpress VEGF, and overexpression of VEGF is associated with increased rates of relapse [18]. The trials planned with bevacizumab in the adjuvant setting will confirm the efficacy of adding an antiangiogenic therapy to standard combinations.

The data from the trials currently ongoing will tell us the efficacy of combining bevacizumab with other drugs used in the metastatic setting, besides docetaxel or capecitabine, the exact role of bevacizumab in neoadjuvant and adjuvant settings, and perhaps it will be possible to identify which patients are most likely to benefit from VEGF-targeted therapies.

\section{References}

1. Foekens JA, et al:: Cancer Res 2001.

2. Relf M, et al.: Cancer Res 1997.

3. Buteau H, et al:: Cancer Res 2002.

4. Guo P, et al.: Cancer Res 2003.

5. Macchiarini P, et al.: Ann Thorac Surg 1994.

6. Lucchi M, et al.: Eur J Cardiothorac Surg 1997.

7. Yuan A, et al.: Int J Cancer Pred Oncol 2000.

8. Gerber $\mathrm{H}$, et al.: Cancer Res 2005.

9. Cobleigh MA, et al.: Semin Oncol 2003.

10. Miller KD, et al.: J Clin Oncol 2005.

11. Relf M, et al.: Cancer Res 1997.

12. Miller KD, et al:: Breast Cancer Res Treat 2005.

13. Russell KS, et al.: Am J Physiol 1999.

14. Pegram M, et al.: Breast Cancer Res Treat 2004.

15. Relf M, et al.: Cancer Res 1997.

16. Lyons JA, et al.: J Clin Oncol 2006.

17. Lyons JA, et al.: J Clin Oncol 2006.

18. Linderholm BK, et al.: Clin Breast Cancer 2003.

\section{S23}

\section{Novel strategies for HER-2-positive metastatic disease: mechanisms and therapeutic options to overcome trastuzumab resistance \\ FJ Esteva}

The University of Texas MD Anderson Cancer Center, Houston, TX, USA

Breast Cancer Research 2007, 9(Suppl 1):S23 (doi: 10.1186/bcr1706)

The HER2 tyrosine kinase receptor is overexpressed in approximately $25 \%$ of invasive breast cancers, and has been associated with a poor prognosis [1]. Trastuzumab (Herceptin) and lapatinib (Tykerb) are the two therapies currently approved by the FDA for the treatment of HER2overexpressing metastatic breast cancer. The majority of patients that initially respond to trastuzumab monoclonal antibody-based therapy develop progressive disease within 1 year of treatment initiation [2]. Preclinical studies have indicated several molecular mechanisms that may contribute to the development of trastuzumab resistance [3]. Increased signaling via the phosphatidylinositol 3-kinase/protein kinase B (or Akt) pathway could contribute to trastuzumab resistance due to activation of multiple receptor pathways. These include HER2-related receptors or non-HER receptors such as the insulin-like growth factor-I receptor, which appears to cross-talk to HER2 in resistant cells [4]. Additionally, loss of function of the tumor suppressor PTEN, the negative regulator of Akt, results in heightened Akt signaling that leads 
to decreased sensitivity to trastuzumab [5]. Decreased interaction between trastuzumab and its target receptor HER2, due to steric hindrance of the HER2 receptor by cell surface proteins, such as MUC4 [6], or by the presence of a truncated HER2 protein [7], may block inhibitory actions of trastuzumab. Novel therapies targeted against these aberrant molecular pathways are being studied in laboratory and clinical settings, and offer hope that the efficacy and duration of response to trastuzumab can be greatly improved.

Lapatinib (Tykerb) is a small molecule tyrosine kinase inhibitor that targets EGFR and HER-2 [8]. Clinical responses were observed in patients with HER2-overexpressing metastatic breast cancer [9]. A recent phase III randomized trial showed that a combination of lapatinib and capecitabine (Xeloda) was superior to capecitabine alone [10], leading to the FDA approval of lapatinib in 2007. Clinical trials are ongoing to determine the role of lapatinib in the frontline setting for metastatic breast cancer in combination with trastuzumab and taxanes, as well as in the neoadjuvant and adjuvant settings.

Other novel strategies being tested in patients with HER2-overexpressing breast cancer include monoclonal antibodies targeting HER2 on different epitopes than trastuzumab (for example, pertuzumab), attaching toxins to trastuzumab (for example, trastuzumab-DM1), Hsp90 inhibitors that degrade the HER-2 protein (for example, 17-AAG), irreversible small molecule tyrosine kinase inhibitors (for example, HKI272), agents directed against IGF-IR and multitarget kinase inhibitors. Indirect approaches include immunotherapy and anti-angiogenic therapy. Clinical trials are evaluating the safety and efficacy of targeted biologic therapies, both as single agents and in combination with other biologics and in combination with standard chemotherapy and endocrine therapy. One of the main challenges is to match the right patient with the right drug(s) at the right time.

References

1. Slamon DJ, Clark GM, Wong SG, Levin WJ, Ullrich A, McGuire WL: Human breast cancer: correlation of relapse and survival with amplification of the HER-2/neu oncogene. Science 1987, 235:177-182.

2. Esteva FJ, Valero V, Booser D, Guerra LT, Murray JL, Pusztai L, Cristofanilli M, Arun B, Esmaeli B, Fritsche HA, et al:: Phase II study of weekly docetaxel and trastuzumab for patients with HER-2-overexpressing metastatic breast cancer. J Clin Oncol 2002, 20:1800-1808.

3. Nahta R, Yu D, Hung MC, Hortobagyi GN, Esteva FJ: Mechanisms of disease: understanding resistance to HER2-targeted therapy in human breast cancer. Natl Clin Pract Oncol 2006, 3: 269-280.

4. Nahta R, Yuan LX, Zhang B, Kobayashi R, Esteva FJ: Insulin-like growth factor-I receptor/human epidermal growth factor receptor 2 heterodimerization contributes to trastuzumab resistance of breast cancer cells. Cancer Res 2005, 65:1111811128.

5. Nagata Y, Lan KH, Zhou X, Tan M, Esteva FJ, Sahin AA, Klos KS, Li P, Monia BP, Nguyen NT, et al.: PTEN activation contributes to tumor inhibition by trastuzumab, and loss of PTEN predicts trastuzumab resistance in patients. Cancer Cell 2004, 6:117127.

6. Nagy P, Friedlander E, Tanner M, Kapanen Al, Carraway KL, Isola J, Jovin TM: Decreased accessibility and lack of activation of ErbB2 in JIMT-1, a herceptin-resistant, MUC4-expressing breast cancer cell line. Cancer Res 2005, 65:473-482.

7. Scaltriti M, Rojo F, Ocana A, Anido J, Guzman M, Cortes J, Di Cosimo S, Matias-Guiu X, Cajal S, Arribas J, Baselga J: Expression of p95HER2, a truncated form of the HER2 receptor, and response to anti-HER2 therapies in breast cancer. J Natl Cancer Inst 2007, 99:628-638.

8. Nahta R, Yuan LX, Du Y, Esteva FJ: Lapatinib induces apoptosis in trastuzumab-resistant breast cancer cells: effects on insulin-like growth factor I signaling. Mol Cancer Ther 2007, 6: 667-674.

9. Burris HA, III, Hurwitz HI, Dees EC, Dowlati A, Blackwell KL, O'Neil B, Marcom PK, Ellis MJ, Overmoyer B, Jones SF, et al.:
Phase I safety, pharmacokinetics, and clinical activity study of lapatinib (GW572016), a reversible dual inhibitor of epidermal growth factor receptor tyrosine kinases, in heavily pretreated patients with metastatic carcinomas. J Clin Oncol 2005, 23: 5305-5313.

10. Geyer CE, Forster J, Lindquist D, Chan S, Romieu CG, Pienkowski T, Jagiello-Gruszfeld A, Crown J, Chan A, Kaufman B, et al:: Lapatinib plus capecitabine for HER2-positive advanced breast cancer. N Engl J Med 2006, 355:2733-2743.

\section{Posters}

\section{P1}

Prognostic factors for the node-negative breast cancers

\section{B-W Park, J-H Kim, K-S Kim, KS Lee}

Yonsei University College of Medicine, Seoul, Korea

Breast Cancer Research 2007, 9(Suppl 1):P1 (doi: 10.1186/bcr1707)

Objective The proportion of node-negative breast cancer patients has been increasing with improvement of diagnostic modalities and early detection. However, there is a $20-30 \%$ recurrence in node-negative breast cancer. To identify the prognostic factors for node-negative breast cancers, we studied the impact of many clinico-pathologic parameters on the outcome of the node-negative breast cancer patients.

Methods The data of 1,110 node-negative breast cancer patients who underwent curative surgery at the Severance Hospital, Yonsei University College of Medicine, were reviewed. The impact of many clinico-pathological parameters on the outcome was investigated. Univariate survival curves for disease-free survival and death were estimated using the Kaplan-Meier method: group differences in survival time were tested by the log-rank test. Multivariate Cox regression analysis was performed to compare and identify independent prognostic factors.

Results The mean age was 47.2 years. The median follow-up was 88 months. Recurrence occurred in 161 patients; 64 patients with locoregional recurrences, 129 with systemic recurrences, and 32 with both. The 5-year overall survival rate was 93.3\%. The rate of locoregional recurrence for a 10-year period was significantly lower in the mastectomy group compared with those in the breast conservation therapy group (94.7\% versus $79.6 \%, P=0.000$ ). No other prognostic factors except age affected locoregional recurrence. There was less systemic recurrence in patients with age greater than 35 , with histologic grade I, and with intraductal components greater than $20 \%$. Thus, the 10-year distant relapse-free survival rates were $87.4 \%$ versus $79.8 \%(P=0.039), 93.5 \%$ versus $85.5 \%(P=0.024)$, and $94.4 \%$ versus $82.0 \%(P=0.007)$, respectively. There was no statistical significance in the other prognostic factors that influence systemic recurrence.

Conclusion Patient age, histologic grade, and presence of intraductal component were identified as independent prognostic factors in nodenegative breast cancer patients.

\section{P2}

Retreatment with trastuzumab in Her2-positive metastatic breast cancer patients: a clinical study F Carabantes-Ocón, E Saez-Lara, L Burgos-Garcia, E Villar-Chamorro, A Casaus-Hazañas, S Luna, C Martínez Unidad de Mama, Hospital Carlos Haya, Málaga, Spain Breast Cancer Research 2007, 9(Suppl 1):P2 (doi: 10.1186/bcr1708)

Objective To study the benefit of trastuzumab in monotherapy or combined with different chemotherapeutic agents in the treatment for Her2+ metastatic breast cancer (MBC) patients after progression on prior trastuzumab therapy. 
Patients and methods The clinical evolution of patients with Her2+ $\mathrm{MBC}$ diagnosed by $\mathrm{IHC}+/ \mathrm{FISH}+$, and treated with trastuzumab in several lines for the metastatic disease, has been studied retrospectively.

Results Twenty-four patients with Her2+ MBC were treated with several regimens containing trastuzumab alone or associated with chemotherapy and/or hormonotherapy. In the first line of treatment 12 RR (50\%), 11 SD (45\%), with a 95\% clinical benefit, was observed. The patients received a second line obtaining 8 RR (33\%), 15 SD $(62 \%)$, with a clinical benefit of $95 \%$. Seventeen patients were treated with a third line, 5 RR (29.4\%) and 11 SD (64\%) being observed, with a clinical benefit of $93 \%$. Seven patients received a fourth line. In these, 2 RR and 4 SD, with a clinical benefit of $85 \%$, were observed. Fifteen of the patients, with RE+, received hormonotherapy plus trastuzumab alone or with chemotherapy in one or more lines of treatment, obtaining 8 RR $(53 \%)$ and 4 SD (26\%), with a clinical benefit of $79 \%$.

Conclusion The association of herceptin with chemotherapy and/or hormonotherapy demonstrates a very active treatment in patients with Her2+ MBC. The benefit seems to continue in patients who already have received treatment with trastuzumab even in more than one regimen.

\section{P3}

\section{Do breast cancer tumours downsize as well as downgrade with neoadjuvant chemotherapy? M Dani', J McDonnell', S Karp'2, V Jaffe ${ }^{1}$}

${ }^{1}$ Department of Surgery (Breast Firm) and ${ }^{2}$ Department of Oncology, Chase Farm Hospital, London, UK

Breast Cancer Research 2007, 9(Suppl 1):P3 (doi: 10.1186/bcr1709)

Objective Neoadjuvant chemotherapy (NC) is increasingly being used for large primary breast carcinomas with the aim of improving breastconservation surgery (BCS) rates. This study was conducted to assess the tumour response following NC.

Methods In this retrospective study over a 4-year period, 61 women with large operable invasive breast cancers $\left(T_{2-4} N_{0-2} M_{0}\right)$, unsuitable for BCS, were consecutively treated with NC (5-FU, epirubicin, cyclophosphamide and Taxotere). Pathological response was monitored, comparing original core biopsy histology with final excisional histology.

Results The mean age of patients was 48.6 years (range $30-70$ ). Of the 61 patients, BCS was achieved in $48(79 \%)$ patients. On the core biopsy, four (6.5\%) patients had grade I cancer, 26 (43\%) had grade II cancer and $31(51 \%)$ had grade III cancer. Final histology showed no invasive cancer in eight (13\%) patients (seven DCIS, and complete pathological response in one patient). In the rest of the patients, four $(6.5 \%)$ had grade I tumours, $26(43 \%)$ had grade II tumours and 23 $(38 \%)$ had grade III tumours. Overall, 14 patients (23\%) showed a decrease in histological grade (see Table 1). Seven patients (11.5\%) had a higher grade than the initial core.

Table 1 (abstract P3)

Initial histology

$$
\text { (core) }
$$

Final histology designation (post NC)

\begin{tabular}{lcccccc}
\hline Grade & $n$ & $\begin{array}{c}\text { No residual } \\
\text { invasive cancer }\end{array}$ & DCIS & Grade I & Grade II & Grade III \\
\hline III & 28 & 1 & 5 & 1 & 4 & 17 \\
II & 29 & 0 & 2 & 1 & 21 & 5 \\
I & 4 & 0 & 0 & 2 & 1 & 1 \\
\hline
\end{tabular}

Conclusion In our series of patients receiving NC for breast cancer, there is not only a significant downsizing (permitting BCS) but also a trend of downgrading of the tumour, and this is seen particularly in poorly differentiated tumours. The higher grade on final histology compared with the core could be due to an unrepresentative core biopsy in large tumours prior to NC.
P4

Saline instillation into the cavity after conservation surgery for breast cancer is a safe way of improving cosmesis

M Dani, T Tahmid, J McDonnell, V Jaffe

Department of Surgery (Breast Firm), Chase Farm Hospital, London, UK

Breast Cancer Research 2007, 9(Suppl 1):P4 (doi: 10.1186/bcr1710)

Objective To demonstrate that saline instillation is a safe and simple procedure for volume replacement after wide local excision in breast surgery.

Methods We performed a pilot study over a 12-month period at the Chase Breast Unit. One hundred and six patients who underwent wide local excision for breast cancer had saline instilled into the surgical cavity at the time of wound closure. This was to maintain the volume and shape of the breast after removal of significant amounts of tissue. We measured the volume instilled and monitored the wound and breast postoperatively at 1 week, 2 weeks and 3 months. As is our normal practice, all patients received perioperative antibiotics.

Results The volume of fluid instilled varied between 30 and $180 \mathrm{ml}$. The weight of tissue removed was in the range of 12-116 g. The fluid was retained within the cavity. However, in one case the wide local excision cavity unexpectedly communicated with the axillary clearance cavity and all the fluid was evacuated spontaneously through the axillary suction drain with a resultant visible reduction in the volume of the breast. There were no complications of saline instillation. In particular, there was no early or late infection in any of the 106 patients. None of the patients reported any additional discomfort or pain. There were no visible abnormalities apart from a subjective enhancement in the shape and volume of the breast. This improvement in shape and volume was maintained for the entire length of assessment (3 months). Conclusion Saline instillation is a simple and safe method of replacing volume after removal of significant amounts of breast tissue. Surprisingly, the benefits seem to persist. We are now proceeding to fully evaluate this technique in a formal prospective trial.

\section{P5}

A safety and efficacy study of bleomycin sulfate and electroporation in patients with metastatic or locally recurrent breast cancer

\section{Paramanov', O Tyurin', S Polenkov' ${ }^{2}$, PM Goldfarb ${ }^{3}$}

${ }^{1}$ Cherkassy Regional Oncologic Clinic, Cherkassy, Ukraine; ${ }^{2}$ Chernigiv Regional Oncology Centre, Chernigiv, Ukraine; ${ }^{3}$ Consulting Medical Director, Inovio Biomedical Corporation, San Diego, CA, USA

Breast Cancer Research 2007, 9(Suppl 1):P5 (doi: 10.1186/bcr1711)

Objective The study evaluated bleomycin sulfate (BS) followed by electroporation (EP) treatment in patients with recurrent in-breast or chest-wall tumors. EP treatment following intralesional injection of BS has been studied in various cancers, and significantly increased destruction of cancer cells within the treatment field. The treatment has also been used as an intracellular delivery system for gene therapies and DNA vaccines.

Methods Patients with histologically confirmed recurrent breast cancer following partial or complete mastectomy received BS intratumorally, $1 \mathrm{unit} / \mathrm{cm}^{3}$ tumor volume, followed by EP under general anesthesia. A CE-marked medical device (MedPulser ${ }^{\circledR}$ ) and a six-needle array applicator were used to electroporate each tumor. Safety evaluations and monitoring for local tumor recurrence were performed periodically up to 24 weeks.

Results Ten female patients with 11 tumors were enrolled. The mean age was 58.0 years (range 43.6-67.7). The mean tumor volume was $1.81 \mathrm{~cm}^{3}(0.01-6.60)$, the mean BS dose was $1.80 \mathrm{U}$ (range 0.50-5.08), and the mean number of EP applications was 11 (range 7-16). No treatment-related serious adverse event was observed. Nonserious adverse events were unremarkable during the 30-day follow-up period. At 24 weeks, the complete response rate was $75 \%(6 / 8)$ among 
patients with evaluations for treated lesions; two patients were inevaluable for tumor response.

Conclusion Treatment with this drug-device combination was well tolerated in these patients, a majority of whom were free of local disease at 24 weeks. These results suggest a promising potential for $\mathrm{BS} / \mathrm{EP}$ as a new treatment for controlling local disease in patients with recurrent breast cancer.

\section{P6}

Predictive value of HER2 expression for the selection of adjuvant chemotherapy in breast cancer patients A Hegmane', U Vikmanis ${ }^{2}$

${ }^{1}$ REH Oncology Center of Latvia, Riga, Latvia; ${ }^{2}$ University of Latvia, Riga, Latvia

Breast Cancer Research 2007, 9(Suppl 1):P6 (doi: 10.1186/bcr1712)

Objective To determine the predictive value of HER2 expression for the selection of adjuvant chemotherapy (antracycline or nonantracycline based) in patients with breast cancer.

Methods Medical records of primary breast cancer patients treated at the Oncology Center of Latvia from January 2002 to August 2005 were retrospectively reviewed. One hundred and ninety-two patients with histopathologically confirmed breast cancer, who had undergone radical surgery and adjuvant chemotherapy (CT), were identified. Tumor characteristics were: ER+ in 62.5\%, ER- in 37.5\%, HER2-positive (3+ by $\mathrm{IHC}$ ) in $30.2 \%$ (58 patients), HER2-negative $(0,1+)$ in 54.7\% (105 patients); patients with score $2+(15.1 \%, 29$ patients) were excluded from analysis. Staging: I (20.8\%), II (51\%), III (29.2\%); 43.8\% patients were node negative, $56.2 \%$ were node positive. Median age at diagnosis was $52.4(30-75)$ years.

Results Antracycline-based CT (FAC or FEC) was received by $62.9 \%$ patients in the HER2-negative group and $41.4 \%$ in the HER2-positive group, nonantracycline based (CMF) CT in $37.1 \%$ and $58.6 \%$ respectively. The median follow-up time was $21.3(6.3-51.7)$ months. A total of $25.2 \%$ tumor recurrences were observed. Median time to relapse was 12.5 (1.4-34) months. In the HER2-positive group the relapse rate was $20.8 \%$ in patients receiving antracycline-based CT versus $29.4 \%$ in patients receiving nonantracycline-based CT $(P=0.09)$. In the HER2-negative group the relapse rate was $33.3 \%$ in patients receiving antracycline-based CT versus $15.8 \%$ in patients receiving nonantracycline-based CT $(P=0.2)$. The subgroup of patients with HER2-negative, ER-negative tumors had the worst prognosis (relapse rate $45 \%$ ) regardless of the CT received.

Conclusion In patients with HER2 overexpression, antracycline-based CT produced a significantly lower relapse rate when compared with nonantracycline-based CT. In HER2-negative patients there was no benefit from antracycline-based CT compared with nonantracyclinebased CT. However, a longer follow-up period is needed to confirm the results.

\section{P7}

\section{Axillary study before surgery in patients with breast} cancer

M Izquierdo, R Fabregas, J Feu, L Lopez Marin, B Navarro,

\section{Ara, M Cusido, F Tresserra}

Institut Universitari Dexeus, Barcelona, Spain

Breast Cancer Research 2007, 9(Suppl 1):P7 (doi: 10.1186/bcr1713)

Objective Axillary study with ultrasound and cytological puncture with fine-needle aspirate (FNA) in patients with invasive breast cancer is a diagnostic method included in protocols.

Methods We studied 159 patients with invasive breast cancer with axillary ultrasound and cytological puncture with fine needle of suspicious nodes before surgery. Suspicious nodes were those with at least one of the following signs: long to short axis ratio less than 1.5, absence of hilius and cortical disruption. If the results were compatible with metastasis, then we performed axillary lymphadenectomy; if it was found to be benign, then we conducted sentinel node biopsy.
Results In 54 patients (33.96\%) FNA was positive. When we conducted axillary lymphadenectomy, 13 patients $(24 \%)$ were found to have one positive node, seven patients (13\%) two positive nodes, nine patients (16\%) three positive nodes, and 25 patients (45\%) more than three positive nodes.

Conclusion Axillary study with ultrasound and FNA before surgery allows exclusion of a group of patients from sentinel node biopsy.

\section{P8}

Resveratrol downregulates acetyl-CoA carboxylase alpha and fatty acid synthase by activating AMP-activated protein kinase and suppressing the mammalian target of rapamycin signal pathway S Yoon, B-W Park, K-S Kim

Yonsei University College of Medicine, Seoul, Korea

Breast Cancer Research 2007, 9(Suppl 1):P8 (doi: 10.1186/bcr1714)

Expression of HER2 is reported to be increased in approximately $30 \%$ of human breast carcinoma. Fatty acid synthase (FASN) was expressed higher in HER2-overexpressing breast cancer cells. Resveratrol (3,5,4'trihydroxystilbene), a red-wine-derived polyphenol, has been shown to suppress cancer cell proliferation, and to interfere with the several signaling pathways and induce apoptosis. We investigated the effects of resveratrol on the expression of lipogenic enzymes in BT-474 cells, in which HER2 overexpresses cells. Resveratrol treatment to BT-474 cells resulted in inhibition of acetyl-CoA carboxylase alpha (ACCalpha) and FASN expression and a strong activation of AMP-activated protein kinase (AMPK), which could be mimicked by the changes caused by AICAR treatment or forced expression of constitutively active AMPK mutant. The decreased ACCalpha and FASN expressions by resveratrol were abolished by overexpression of dominant-negative AMPK mutant. The activation of AMPK was accompanied with the reduction of the mammalian target of rapamycin (mTOR), which plays a key role in the upregulation of ACCalpha and FASN expression in BT-474 cells. These results indicate that downregulation of HER2mediated ACCalpha and FASN expression by resveratrol is regulated through suppressing the mTOR signaling pathway resulting from the activation of AMPK in breast cancer cells.

\section{P9}

Partial breast irradiation with an electron beam for patients submitted to conservative surgery MAC Maia, MM Netto, MS Maciel, JC Donoso, JV Salvajoli, ACA Pellizzon, RC Fogaroli, KW Bocalletti, C Tanous Hospital AC Camargo, São Paulo - SP, Brazil

Breast Cancer Research 2007, 9(Suppl 1):P9 (doi: 10.1186/bcr1715)

Objective To evaluate the feasibility, acute complications and cosmetic results of partial breast cancer radiotherapy in initial tumors, with an electron beam, in patients submitted to conservative surgery and sentinel lymph-node dissection.

Methods From 2005 to 2007 we evaluated 40 patients, median age 63 years, with $\mathrm{T} 1-\mathrm{T} 2$ (maximum $3 \mathrm{~cm}$ ) breast cancer, clinically NO axilla, submitted to conservative surgery and intraoperative radiotherapy with an electron beam.

Results Of 40 patients, we had 73\% T1 and 27\% T2; $82 \%$ NO, 8\% N1micro, and $10 \% \mathrm{~N} 1$. As for acute complications we had $2 \%$ infection and $5 \%$ fat necrosis. Regarding cosmetic results, $85 \%$ were good and excellent, $10 \%$ were fair and $5 \%$ were bad.

Conclusion This is a quite feasible method for partial breast irradiation, with satisfactory results in terms of acute complications and cosmetic results. For local control and late complications we still have to wait longer and wait for the reports of the open trials. It also should be emphasized that these patients should be treated only in a study protocol. 


\section{P10}

Positive axillary lymph node metastases in T1-T3 breast cancer: prognostic value of extracapsular extension

S Maksimovic', Z Gojkovic ${ }^{2}$, M Opric ${ }^{3}$

${ }^{1}$ General Hospital "Sveti Vracevi" in Bijeljina, Bosnia and Herzegovina; ${ }^{2}$ Clinic for Oncology Clinical Center Banja Luka, Bosnia and

Herzegovina; ${ }^{3}$ Clinical Center "Bezanijska Kosa" Belgrade, Serbia

Breast Cancer Research 2007, 9(Suppl 1):P10 (doi: 10.1186/bcr1716)

Background Extracapsular extension (ECE) of axillary metastases has importance as a risk factor for local or distant recurrence. Poorer survival in breast cancer has been suggested, but its prognostic value has not been uniformly confirmed.

Methods From January 2000 to March 2007, 356 breast cancer patients were operated on in the Department of General Surgery of General Hospital 'Sveti Vracevi' in Bijeljina. We selected 173 (48.6\%) cases with pT1-pT3 node-positive breast cancer. The prognostic significance of ECE of axillary metastases was evaluated with respect to disease-free survival, overall survival, and the patterns of disease recurrence. Such prognostic significance was then compared with that of other clinical and pathologic factors.

Results Ninety-five patients (26.68\%) presented with ECE. Thirty patients $(31.57 \%)$ were identified as having three or less lymph nodes involved, 26 patients $(27.36 \%)$ patients four to six nodes, 18 patients (18.9\%) seven to nine nodes, and $22.16 \%$ patients 10 or more nodes, respectively. With a median follow-up of 86 months, factors with independent prognostic value for disease-free survival by multivariate analysis included absence of estrogen receptors $(P<0.005), \mathrm{pN}$ category $(P<0.01)$, presence of lymphovascular invasion (LVl; $P<0.005)$, and ECE $(P<0.001)$. An independent negative prognostic effect on overall survival was observed for absence of estrogen and progesterone receptors $(P<0.05), \mathrm{pN}$ category $(P<0.05)$, and presence of LVI $(P<0.005)$ and ECE $(P<0.001)$.

Conclusion ECE demonstrated a stronger statistical significance in predicting prognosis than the $\mathrm{pN}$ category and was also related to an increased risk of distant recurrences. We suggest that the decision on adjuvant therapy should consider the presence of ECE of axillary metastases and peritumoral LVI as indicators of high biological aggressiveness. Balancing the risks and benefits of irradiation, we continue to recommend that complete axillary irradiation is not routinely indicated after adequate axillary dissection.

\section{P11}

\section{Study of the combination gemcitabine and trastuzumab in the treatment of HER2+ metastatic breast cancer \\ S Menjón-Beltrán, R Olivencia-Dueso, E González-Jiménez, MJ Titos}

Unidad de Ginecología Oncológica, Hospital Universitario Virgen de las Nieves, Granada, Spain

Breast Cancer Research 2007, 9(Suppl 1):P11 (doi: 10.1186/bcr1717)

Objective Trastuzumab and gemcitabine are two active drugs for metastatic breast cancer $(\mathrm{MBC})$ treatment. We conducted a retrospective study of this combination in patients with Her2+ MBC in our hospital.

Patients and methods Retrospective assessment of the efficacy of trastuzumab and gemcitabine association in patients with MBC previously treated with different lines of chemotherapy.

Results Seventeen heavily pretreated patients with poor prognosis were evaluated. Fourteen patients $(82 \%)$ had received prior adjuvant chemotherapy and 11 patients (64\%) prior adjuvant radiotherapy; 12 patients $(70 \%)$ had received prior chemotherapy for MBC. All the patients had received taxanes and $15(88 \%)$ had received anthracyclines also. We observed a $41 \%$ rate of response, two complete responses, five partial responses, and $41 \%$ stable disease, obtaining $82 \%$ clinical benefit. The time-to-progression was 7.2 months. Median survival has not yet been reached. Nine of the patients had received previous treatment with trastuzumab alone or in combination, four additional responses and four stable disease responses being observed, which produces an $88 \%$ clinical benefit. One patient discontinued treatment because of LVEF decline (>50\%).

Conclusion Trastuzumab and gemcitabine constitutes a well-tolerated and very active regimen for Her2+ MBC heavily pretreated patients, including patients who have been previously treated with trastuzumab.

\section{P12}

\section{Clinical role of trastuzumab in metastatic breast} cancer: experience of a center

\section{S Menjón-Beltrán, R Olivencia, E Gonzalez}

Unidad de Ginecología Oncológica, Hospital Universitario Virgen de las Nieves, Granada, Spain

Breast Cancer Research 2007, 9(Suppl 1):P12 (doi: 10.1186/bcr1718)

Objective To study the efficacy of different regimens of treatment based on trastuzumab in patients with Her2+ metastatic breast cancer (MBC).

Patients and methods Medical records of 47 Her2+ MBC patients were retrospectively studied in our center between December 1999 and February 2004. The Her2 status was determined by immunohistochemistry (Herceptest), FISH being used to discriminate the doubtful cases.

Results Forty-seven patients with MBC of bad prognosis treated previously have been evaluated, a complete response $(C R)$ being observed in $30 \%$ of the cases with a response rate (RR) (CR + partial response (PR)) of $63 \%$. The stable disease rate (SD) was $12 \%$, which provides a clinical benefit $(C R+R P+S D)$ of $75 \%$. Thirteen of these patients received a new scheme of treatment based on trastuzumab after progression to the first regimen containing trastuzumab, $76 \%$ of $\mathrm{RR}(\mathrm{CR}+\mathrm{PR})$ and $30 \%$ of CR being observed. Three of these patients received a third regimen of treatment with trastuzumab, still obtaining one CR (RR 33\%), and one SD.

Conclusion The association of trastuzumab with chemotherapy constitutes a very active regimen in previously treated patients with MBC of poor prognosis. This activity even continues in patients who have already received a previous treatment based on trastuzumab.

\section{P13}

\section{BRCA1a has antitumor activity in triple-negative breast cancers}

\section{Yuli, N Shao, G Oprea-Ilies, J Okoli, ESP Reddy, VN Rao}

Cancer Biology Program, Department OB/GYN, Morehouse School of Medicine, Atlanta, GA, USA

Breast Cancer Research 2007, 9(Suppl 1):P13 (doi: 10.1186/bcr1719)

Most BRCA1-related breast cancers are high-grade, basal-like, estrogen receptor (ER)-negative, progesterone receptor (PR)-negative, and Her2-negative. Triple-negative breast cancers are more common in younger black women than their white counterparts, have higher rates of distant metastasis, and currently there are no effective treatments against these cancers. We have previously characterized a splice variant of BRCA1 (BRCA1a/p110), which is expressed at reduced levels in several breast tumors. Stable expression of BRCA1a resulted in inhibition of growth in vitro of human breast, ovarian, prostate and colon cancer cells, and only those cells with wild-type $\mathrm{Rb}$ were sensitive to BRCA1a-induced growth suppression and the status of p53 did not affect the ability of BRCA1a to suppress growth of tumor cells. We have introduced BRCA1a into the CAL51 cell line, which is negative for $E R$ and $P R$, and our results using semiquantitative immunocytochemistry show CAL51 to be negative for HER2. These transfectants were analyzed for BRCA1a protein expression by western blot analysis. The BRCA1a transfectants were slow growing and $98 \%$ inhibited in their growth in soft agar. BRCA1a also significantly inhibited CAL-51 triple-negative breast cancer xenografts in nude mice. These results suggest that the majority of exon 11 sequences lost in BRCA1a are not required for the tumor suppressor 
function. This is the first report showing antitumor activity of BRCA1a in triple-negative breast cancers.

\section{P14}

\section{Diagnosis of familial breast cancer by multiplex PCR and clinical parameters H Rassi}

National Medical Academy of Post-Graduate Education, Kiev, Ukraine Breast Cancer Research 2007, 9(Suppl 1):P14 (doi: 10.1186/bcr1720)

Objective Mutation analysis of mtDNA and nDNA are helpful in the determination of developmental potential, early diagnosis and gene therapy for breast cancer. In our study, we optimized the multiplex PCR in AFPBT and compared the results with morphological and immunohistochemical parameters for detection of BRCA mutations and mtDNA4977 deletion in familial and nonfamilial breast cancers.

Methods The multiplex PCR was conducted on DNA from 71 archive breast tissue samples and 13 blood samples. We compared different paraffin removal procedures and DNA extraction procedures in combination in order to optimize and compare the results of the multiplex PCR with morphological and immunohistochemical parameters for diagnosis of breast cancers.

Results The highest proportion of successful gene amplifications was obtained with a DNA isolation procedure using proteinase $\mathrm{K}$ digestion with followed boiling. Three 5382insC mutations were identified from 16 archival familial patients (19\%) and five mtDNA4977 deletions were detected from nine blood familial breast cancers by the multiplex PCR. The mtDNA4977 deletion was highly prevalent in peripheral blood $(56 \%)$, but it was absent in the breast tissue of cancer cases. Furthermore, familial breast cancer tumors exhibited higher mitotic activity, higher polymorphism, lower necrosis, lower tubules, higher ERnegatives and PR-negatives and lower TP53-positives than nonfamilial cancers.

Conclusion Our results demonstrate that DNA extracted by the simple boiling method with a microwave yielded higher proportions of successful gene amplifications than the DNA extraction kit. Furthermore, differences in successful gene amplification may be related to the size and number of the gene fragments amplified. Our analysis shows that testing of mtDNA4977 deletions and 5382insC in combination with morphological and immunohistochemical parameters may be an extremely effective and inexpensive tool in testing breast cancer patients aimed to identify individuals with high risk of familial breast cancers.

\section{P15}

\section{Novel method of sentinel lymph node detection in} malignant tumors using preparation 'UNIMAG'

\section{B Surguladze, R Zhorzoliani, T Tskitishvili}

"Magnetic Fluids in Medicine and Biology", ATT Ltd, Georgia, USA Breast Cancer Research 2007, 9(Suppl 1):P15 (doi: 10.1186/bcr1721) Preparation 'UNIMAG' is registered by the pharmacological committee of Georgia (Cert.: DA Nr-000142, 08.04.2005; Author - PhD, ScD, Professor Besiki Surguladze; Owner - 'ATT' Ltd, Sci. Laboratory 'Magnetic fluids in Medicine and Biology'). Preparation 'UNIMAG' represents a magnet-sensitive stabile suspension of magnetite nanoparticles (magnetic fluid). After the peritumoral injection of 'UNIMAG', magnetite nanoparticles will be absorbed by macrophages, which deliver them through the lymphatic capillaries to regional lymph nodes. This mechanism of magnetite nanoparticle transport underlies the new method of sentinel lymph node detection. Diversely to the other dyestuff agents used in this direction, magnetite nanoparticles actively filled lymphatic nodes and colored them in bold black. This significantly facilitates the atraumatic separation of lymph nodes during lymphadenectomy. Besides the intraoperative indication of lymphatic nodes, 'UNIMAG' provides the possibility of X-ray and ultrasound imaging of sentinel lymph nodes and performing their biopsy in the preoperative period. This assuredly helps in a better planning of the operation and considerably improves its radicalism. Even in the case of ideal surgical intervention it is impossible to remove all the microscopic lymphatic vessels and nodes that could be damaged by the tumor micrometastasis. In the field of high-frequency electromagnetic waves, the magnetite nanoparticles give us possibilities of local, distance hyperthermia on micrometastatic lesions. This minimizes the potentiality of development of postoperative recurrence and secondary metastatic processes.

\section{P16}

Frequency of febrile neutropenia in patients treated with taxane/epirubicine and colony-stimulating growth factors for breast cancer: comparison of filgrastim/lenograstim with pegfilgrastim W Schippinger, R Holub, N Dandachi, $\mathbf{T}$ Bauernhofer Hellmut Samonigg Medical University Graz, Department of Internal Medicine, Division of Medical Oncology, Graz, Austria

Breast Cancer Research 2007, 9(Suppl 1):P16 (doi: 10.1186/bcr1722) Objective This report describes a single centre's experience on the efficacy of pegfilgrastim compared with filgrastim or lenograstim in reducing the incidence of febrile neutropenia in patients receiving combination chemotherapy with docetaxel or paclitaxel and epirubicin in neoadjuvant and adjuvant settings.

Methods A total of 118 patients with breast cancer were treated with either epirubicin $75 \mathrm{mg} / \mathrm{m}^{2}$ and docetaxel $75 \mathrm{mg} / \mathrm{m}^{2}$ or with epirubicin $90 \mathrm{mg} / \mathrm{m}^{2}$ and paclitaxel $200 \mathrm{mg} / \mathrm{m}^{2}$ every 3 weeks; 88 patients received G-CSF support with daily filgrastim or lenograstim and 30 patients with pegfilgrastim once per cycle.

Results Eight $(9.1 \%)$ patients with prophylactic filgrastim or lenograstim support developed febrile neutropenia, and one (3.3\%) patient in the pegfilgrastim group $(P=0.445)$. Febrile neutropenia occurred in $13(2.7 \%)$ of 476 filgrastim or lenograstim supported chemotherapy cycles, and in two (1.2\%) of 172 cycles with pegfilgrastim support $(P=0.376)$. The frequency of chemotherapy delays and dose reductions was not significantly different between the two G-CSF treatment groups.

Conclusion The results of this study demonstrate a trend towards superiority of pegfilgrastim over filgrastim or lenograstim in reducing the frequency of febrile neutropenia in patients treated with taxane and epirubicin chemotherapy regimens for breast cancer.

\section{P17}

Comparison of side-effect profiles during active treatment versus follow-up in the International Breast Cancer Intervention Study I tamoxifen prevention trial

I Sestak', R Edwards' ${ }^{1}$, A Howell' ${ }^{2}$, J Cuzick'

${ }^{1}$ Cancer Research UK, Centre for Epidemiology, Mathematics and Statistics, London, UK; ${ }^{2}$ Department of Surgical Oncology, Newcastle Mater Hospital, University of Newcastle, UK

Breast Cancer Research 2007, 9(Suppl 1):P17 (doi: 10.1186/bcr1723) Objective Tamoxifen is an effective drug but its role in prevention is limited by its side-effect profile, particularly related to endometrial problems and thrombotic events. We present updated results on the comparison of side effects between active treatment and the follow-up period incorporating four additional years of follow-up.

Methods In the International Breast Cancer Intervention Study I (IBIS-I) study, 7,154 women at increased risk of breast cancer were either randomised to tamoxifen $20 \mathrm{mg} /$ day or placebo for 5 years. Women gave detailed information of any side effects at each 6-monthly followup visit and once a year after active treatment.

Results During active treatment, large numbers of side effects were reported by participants in both treatment arms. However, the only major categories that showed differences were vasomotor and gynaecological side effects, which were about $12 \%$ higher in the tamoxifen group than the placebo group. Thromboembolic events were significantly higher in the tamoxifen group compared with the placebo 
group (85 versus $42, P<0.001$ ), whereas myocardial infarctions were reduced in women on tamoxifen during active treatment ( 2 versus 7 , $P=0.09$ ). Women on tamoxifen developed significantly more endometrial cancer during active treatment than women on placebo (12 versus $3, P=0.02)$. After active treatment, most menopausal-like side effects decreased and the differences between the two treatment arms were not significant. Reports on vaginal discharge decreased after stopping tamoxifen. Thromboembolic events were similar between the two treatment arms after active treatment (32 versus $26, P=0.4$ ). No significant difference in endometrial cancer between the two treatment groups was found during follow-up time ( 8 versus $5, P=0.4$ ).

Conclusion Although large numbers of side effects were reported, tamoxifen was well tolerated and no new safety concerns were identified. For most side effects no significant difference between treatment groups was found after ceasing tamoxifen.

\section{P18}

Minimal residue disease (MRD)

A Zaidi ${ }^{1}$, G Tripuraneni ${ }^{1}$, S Weller ${ }^{2}$, B Ward ${ }^{2}$, HD Sinnett ${ }^{3}$, RC Coombes ${ }^{1}$, MJ Slade ${ }^{1}$

${ }^{1}$ Department of Oncology, Imperial College, London UK, ${ }^{2}$ Department of Medical Oncology and ${ }^{3}$ Department of Surgery, Charing Cross Hospital, London UK

Breast Cancer Research 2007, 9(Suppl 1):P18 (doi: 10.1186/bcr1724) Introduction: We developed a quantitative PCR (QPCR) technique for the detection of cytokeratin 19 (CK19) transcripts in blood and bone marrow and compared this with immunocytochemistry (ICC). The aim was to monitor these assays for micrometastases in primary breast cancer patients.

Materials and methods Bone marrow (BM) aspirates were taken 2-4 years following chemotherapy from 39 patients who had entered a trial comparing high-dose chemotherapy $(n=17)$ with standard chemotherapy $(n=22)$. The BM was tested for the presence of CK19positive cells using ICC and OPCR. We compared this with 20 lowrisk (T1No) patients.

Results See Table 1. A total of 232 aspirates were analysed from the high-risk patients and 100 from the T1NO patients. The number of patients positive was therefore approximately the same irrespective of therapy in the high-risk patients, and the levels of positivity were not significantly different (PCR, $P=0.078$; and ICC, $P=0.253$ ). The levels of positivity, however, were higher in the high-risk patients than in the low-risk patients.

Table 1 (abstract P18)

\begin{tabular}{|c|c|c|c|c|}
\hline & $\begin{array}{c}\text { Standard } \\
\text { chemotherapy } \\
(n=22)\end{array}$ & $\begin{array}{c}\text { High-dose } \\
\text { chemotherapy } \\
(n=17)\end{array}$ & $\begin{array}{l}\text { All high risk } \\
\quad(n=39)\end{array}$ & $\begin{array}{c}\text { T1N0 } \\
(n=20)\end{array}$ \\
\hline QPCR & $58 / 138(42 \%)$ & $41 / 94(44 \%)$ & $103 / 238(43 \%)$ & $26 / 100(26 \%)$ \\
\hline ICC & $50 / 110(45 \%)$ & $37 / 80$ (46\%) & $90 / 198$ (45\%) & $27 / 96(28 \%)$ \\
\hline Both & $34 / 110$ (34\%) & 26/80 (32\%) & $44 / 198(22 \%)$ & $14 / 96(15 \%)$ \\
\hline Either & $73 / 138(53 \%)$ & $42 / 94(45 \%)$ & $127 / 238(53 \%)$ & $39 / 100$ (39\%) \\
\hline
\end{tabular}

Conclusion We have demonstrated that: (a) patients who received high-dose and standard chemotherapy showed similar results, (b) patients with a low-risk of relapse had markedly lower values as compared with high-risk primary breast cancer patients, and (c) micrometastases persist in a substantial proportion of patients.

\section{Proffered Papers}

\section{P19}

Algorithm for identification of individuals with hereditary predisposition to breast cancer and other breast cancer-associated forms of malignant neoplasms

\section{Zalutsky, NN Antonenkova, GM Porubova}

NN Alexandrov Research Institute of Oncology and Medical Radiology, Minsk, Belarus

Breast Cancer Research 2007, 9(Suppl 1):P19 (doi: 10.1186/bcr1725)

Introduction Hereditary cancers make up $5-15 \%$ of all forms of breast cancer (BC). BRCA $1 / 2$ mutations determine about $50 \%$ of hereditary $\mathrm{BC}$ forms and other pathologic BRCA1/2 genotype-associated forms of malignant neoplasms. Meta-analysis has demonstrated a high probability for development of ovarian cancer, gastric cancer, colon cancer, endometrial cancer, pancreatic cancer, skin melanoma, urinary bladder cancer, and head and neck tumours with pathologic BRCA1/2 genotype, which should be taken into consideration in medicogenetic consulting and monitoring of patient carriers.

Objective To develop and introduce into clinical practice a diagnostic algorithm targeted at active identification of hereditary BC and other BRCA1/2 mutation-associated forms of malignant neoplasms.

Methods Questionnaires for patients, and molecular genetic blood analysis.

Results Questionnaires for patients with BC, ovarian cancer or colon cancer reveal individuals with cancer-burdened heredity, whose blood is analysed for the presence of germinal mutations. Relatives of BRCA1/2 mutation-positive patients are subject to examination for both germinal mutation-carrying and tumour markers. A cohort is formed for targeted prophylactic medical examinations.

Conclusion (1) An original method is developed for active identification of hereditary BC and other BC-associated malignant neoplasms. (2) The proposed method makes it possible to conduct targeted and efficient prophylatic medical examinations of germinal mutation carriers.

\section{P20}

\section{Identification of individuals with hereditary} predisposition to breast cancer and other breast cancer-associated forms of malignant neoplasms: data of the Belarusian Cancer Registry

\section{Zalutsky, NN Antonenkova}

NN Alexandrov Research Institute of Oncology and Medical Radiology, Minsk, Belarus

Breast Cancer Research 2007, 9(Suppl 1):P20 (doi: 10.1186/bcr1726)

Introduction Nowadays, all developed countries spend enormous resources on breast cancer (BC) treatment. However, no significant reduction in the population's BC rates due to the improvement of treatment methods can be expected in the near future.

Objective To use the data of the Belarusian Cancer Registry (BCR) for active identification of individuals with hereditary predisposition to $\mathrm{BC}$ and other BC-associated forms of malignant neoplasms.

Materials and methods To make the analysis, 4,442 patients with second primaries (SP), one of which was BC, were selected, as well as $\mathrm{BC}$ patients with disease origination before age 40 . There were 7,137 such patients.

Results BC was most commonly associated with skin cancer (24.9\%), endometrial cancer $(11.8 \%)$, ovarian cancer $(9 \%)$, gastric cancer (7.8\%), cervical cancer $(5.9 \%)$, rectum cancer $(5.2 \%)$, thyroid cancer $(5 \%)$, colon cancer $(4.8 \%)$, renal cancer $(3.6 \%)$, lung cancer $(3.4 \%)$, skin melanoma $(2.3 \%)$ and other cancers $(16.3 \%)$. The fate of each patient was retraced.

Conclusions (1) BCR data enable one to identify individuals potentially predisposed to hereditary $\mathrm{BC}$ and other $\mathrm{BC}$-associated forms of malignancies, according to two features. (2) Using the risk factors for 
development of hereditary BC and other BC-associated forms of malignant neoplasms makes it possible to draw the relatives of the identified individuals into genetic consulting. (3) The rates of BC association with other forms of malignancies in SP patients were evaluated in the Belarusian population. (4) The use of BCR data and active involvement of relatives of persons with a cancer burden in oncogenetic consulting offer opportunities for early diagnosis and prevention of hereditary $\mathrm{BC}$ and other $\mathrm{BC}$-associated forms of malignant neoplasms.

\section{P21}

\section{Tachosil $^{\circledR}$ to reduce the morbidity of axillary lymph} node dissection in breast cancer

\section{E Barranger, O Morel, G Akerman, C Malartic, D Clement}

Département de Gynecologie-Obstétrique, Hôpital Lariboisière, Paris, France

Breast Cancer Research 2007, 9(Suppl 1):P21 (doi: 10.1186/bcr1727)

Background Seroma formation after axillary lymph node dissection in breast cancer remains a problem despite many efforts to reduce surgery-related morbidity. The aim of the present study was to evaluate the impact of a fibrin-glue-coated collagen patch (Tachosil ${ }^{\circledR}$; Nycomed Laboratory, Denmark) to reduce the volume and duration of postoperative axillary drainage, the duration of hospital stay, and procedural safety.

Patients and methods Twenty-five patients with breast cancer were included in this prospective study. All patients underwent breast surgery including lumpectomy or mastectomy followed by axillary lymph node dissection (Berg Level I and II). In 13 patients, a surgical haemostasis sponge Tachosil ${ }^{\circledR}$ was applied from the apex axillae to the thoracic longus nerve and 12 had standard closure of the axillary lymphadenectomy area. Patients did not differ with respect to general characteristics, such as age, body mass index, treatment modality, and tumor stage distribution. There were six mastectomy and five lumpectomy operations in the Tachosil ${ }^{\circledR}$ group, and seven mastectomy and four lumpectomy surgeries in the control group. The mean number of removed lymph nodes was 11.9 in the Tachosil ${ }^{\circledR}$ group with a mean of 1.2 metastatics nodes, and was 12.2 in the control group with a mean of 2.5 metastatic nodes.

Results The mean duration of axillary drainage was 3.3 days in the Tachosil ${ }^{\circledR}$ group and 5.5 days in the control group. The mean total drainage volume was $246 \mathrm{ml}$ in the Tachosil ${ }^{\circledR}$ group and $470 \mathrm{ml}$ in the standard closure group. The mean length of postoperative hospital stay was 3.5 days in the Tachosil ${ }^{\circledR}$ group and 5.5 days in the standard closure group. Seroma formation after drain removal was found in four patients with a mean of drainage volume of $161 \mathrm{ml}$ in the Tachosil ${ }^{\circledR}$ group, and in five patients with a mean of drainage volume of $517 \mathrm{ml}$ in the standard closure group. No intolerance of the product was described.

Conclusion The use of Tachosil ${ }^{\circledR}$ in the axillary area in patients with breast cancer requiring axillary dissection seems to reduce the volume and duration of postoperative axillary drainage, the duration of hospital stay, and the frequency and volume of seroma after axillary drainage. Larger series are required to confirm these results.

\section{P22}

First clinical results on the potential of intraoperative imaging for sentinel lymph node biopsy in breast cancer

E Barranger ${ }^{1}, K_{\text {Kerrou}}^{2}$, S Pitre ${ }^{3}$, MA Duval ${ }^{3}$, C Coutant $^{1}$, R Siebert ${ }^{3}$, J Talbot' ${ }^{2}$, Y Charon ${ }^{3}$, S Uzan ${ }^{1}$

${ }^{1}$ Gynécologie Obstétrique, Hôpital Lariboisière, Hôpital Tenon AP-HP, Paris, France; ${ }^{2}$ Médecine Nucléaire, Hôpital Tenon AP-HP, Paris, France; ${ }^{3}$ Laboratoire IMNC-CNRS Paris 7-Paris 11, Orsay, France

Breast Cancer Research 2007, 9(Suppl 1):P22 (doi: 10.1186/bcr1728)

Background The growing interest in the sentinel lymph node (SLN) detection concept has led to the development of several prototypes of hand-held gamma cameras. After the first successful clinical trials with

\section{Figure 1 (abstract P22)}

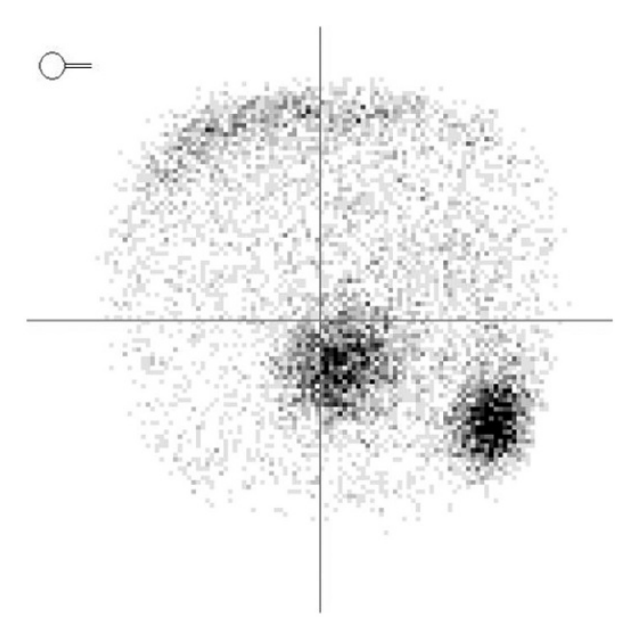

Preoperative image by $\mathrm{POCl}$ showing two hot sentinel nodes.

Table 1 (abstract P22)

Results of SLN identification with lymphoscintigraphy and POCI

\begin{tabular}{lcccc}
\hline & Total SLNs & Mean & $95 \% \mathrm{Cl}$ & Range \\
\hline Preoperative lymphoscintigraphy & 54 & 1.6 & $1.28-1.99$ & $0-4$ \\
POCl camera the day before surgery & 59 & 1.8 & $1.46-2.12$ & $0-4$ \\
POCl intraoperative imager & 61 & 1.9 & $1.55-2.15$ & $0-4$ \\
\hline
\end{tabular}

intraoperative imaging probes, the time has come to quantify the clinical benefit of these devices.

Objective To compare the hand-held camera (POCl) with a lymphoscintigraphy in order to precisely localize SLNs in patients with breast cancer requiring SLN biopsy.

Methods The SLN protocol consisted of a peritumoral injection of $160 \mathrm{MBq}{ }^{99 m} T \mathrm{c}$-labeled nano-colloids performed 2 hours before the lymphoscintigraphy, which is realized preoperatively using a conventional single-head gamma camera and then the hand-held $\mathrm{POCl}$ camera (field-of-view, $13 \mathrm{~cm}^{2}$ ) (Figure 1). The day after, in the operating room, the patients undergo one blue-dye subcutaneous tumoral injection. Before incision the surgeon first uses the $\mathrm{POCl}$ to perform an intraoperative lymphoscintigraphy and then the counting probe (Europrobe Csl, Eurorad) for transcutaneous SLN prelocalizations. The $\mathrm{POCl}$ camera was slowly moved on the skin until SLN identification. The axillary area was scanned with the POCl camera with 10-second acquisition images. SLN detection was defined as positive on the basis of a local radioactivity increase in the intraoperative images of the surveyed region. The SLN excision biopsy was guided, following the established procedure, using the counting probe and blue dye. Finally, a complementary scanning procedure was performed with the $\mathrm{POCl}$ directly in the operative wound before skin closure to confirm complete excision or to detect some residuals SLNs. The number and the localization of SLNs obtained by both intraoperative detectors are compared with those obtained the day before with the conventional gamma camera.

Results To date, 33 patients (mean age: 64 years) have been included in this clinical protocol. A SLN was identified by lymphoscintigraphy in $31 / 33$ patients $(94 \%)$, versus $30 / 33(90.9 \%)$ with the $\mathrm{POCl}(P>0.3)$. The mean number of SLNs identified by lymphoscintigraphy was 1.6 (range: $0-4$ ), versus 1.8 (range: $0-4$ ) with the $\mathrm{POCl}$ (Table 1). The day of the surgery, at least one SLN (mean: 1.9; range: 0-4) was identified 
by the $\mathrm{POCl}$ in $32 / 33$ patients $(97 \%)(P=0.13)$. At least one SLN (mean: 2.3; range: 1-4) was identified in all patients during the surgery. Concerning the duration of these procedures, the mean acquisition time was 15 minutes (range: 10-30) with lymphoscintigraphy, 8.8 minutes (range: $3-18$ ) with the $\mathrm{POCl}$ used the day before surgery and 3.35 minutes (range: $2-6.5$ ) with preoperative $\mathrm{POCl}$.

Conclusion These preliminary clinical results show that the $\mathrm{POCl}$ camera is able to predict the number and localization of breast cancer $\mathrm{SLNs}$. The $\mathrm{POCl}$ camera appears to be a promising tool to complete or replace the preoperative standard lymphoscintigraphy, especially in surgical centers without an onsite nuclear medicine department.

\section{P23}

\section{Preliminary safety data of preoperative} chemotherapy plus trastuzumab, lapatinib or both in HER2-positive operable breast cancer

V Guarneri ${ }^{1}$, A Frassoldati' ${ }^{1}$ F Piacentini' ${ }^{1}$ K Cagossi ${ }^{2}$, L Cavanna ${ }^{3}$, A Michelotti ${ }^{4}$, G Jovic ${ }^{1}$, S Giovannelli ${ }^{1}$, G Ficarra ${ }^{5}$, C Oliva ${ }^{6}$, P Conte $^{1}$

${ }^{1}$ Department of Oncology and Hematology, University of Modena and Reggio Emilia, Modena, Italy; ${ }^{2}$ Division of Medical Oncology Ramazzini Hospital, Carpi, Italy; ${ }^{3}$ Division of Medical Oncology, Hospital of Piacenza, Italy; ${ }^{4}$ Division of Medical Oncology, S. Chiara University Hospital, Pisa, Italy; ${ }^{5}$ Department of Pathology, University of Modena and Reggio Emilia, Modena, Italy; ${ }^{6}$ Oncology Medicine Development Center, GlaxoSmithKline, Greenford, UK

Breast Cancer Research 2007, 9(Suppl 1):P23 (doi: 10.1186/bcr1729) Introduction and objective Lapatinib $(\mathrm{L})$ is a tyrosine kinase inhibitor of EGFR and HER2. The inhibition of these two pathways can affect tumor growth by reducing the EGFR-dependent proliferative stimulus, by restoring apoptosis, and possibly by enhancing sensitivity to chemotherapy (CT). On these premises, the combination of $L$ with CT or with $\mathrm{CT}$ and trastuzumab $(\mathrm{T})$ is promising. We have therefore designed a phase II randomized trial to evaluate the activity and safety of this combination as preoperative therapy for HER2+ operable breast cancer (BC). The primary endpoint was percentage of pathologic complete response $(\mathrm{pCR})$. Secondary aims were the breast objective response, breast conservative surgery, safety, molecular responses, and gene expression related to $\mathrm{pCR}$.

Patients and methods After a core biopsy for diagnosis, biomarker evaluation and storage of fresh tissue for molecular analyses, patients with HER2+ stage II-IIIA BC are randomized to: $\operatorname{arm~A,~CT~+~T;~arm~B,~}$ $\mathrm{CT}+\mathrm{L} ; \operatorname{arm~C}, \mathrm{CT}+\mathrm{T}+\mathrm{L}$. CT consists of: paclitaxel $80 \mathrm{mg} / \mathrm{m}^{2}$ weekly for 12 weeks, followed by four courses of FEC (5-FU $600 \mathrm{mg} / \mathrm{m}^{2}+$ epirubicin $75 \mathrm{mg} / \mathrm{m}^{2}+$ cyclophosphamide $600 \mathrm{mg} / \mathrm{m}^{2}$ i.v.) every 3 weeks. T is administered at the dose of $2 \mathrm{mg} / \mathrm{kg}$ weekly in arms A and $C ; L$ is administered at $1,500 \mathrm{mg}$ p.o. daily in $\operatorname{arm} B$, and at $1,000 \mathrm{mg}$ p.o. daily in arm C. Both $T$ and $L$ are administered throughout the duration of CT. The following biomarkers are evaluated at baseline, and at surgery: EGFR, HER2, pTEN, pAKT, pMAPK, apoptosis (TUNEL Test), Ki67.

Sample size Assuming a 50\% increase in the pCR rate for the CT $+\mathrm{T}+$ $L$ arm versus $C T+T$ or $C T+L, 52$ patients will be enrolled in the first stage; in case we observe $13 \mathrm{pCR}$, an additional 68 patients will be enrolled, for a total of 120 patients (Simon's design).

Results Twelve patients have so far been randomized. Preliminary safety data will be presented at the meeting.

Acknowledgement Supported by GlaxoSmithKline.

\section{P24}

\section{Significance of nuclear medicine: ${ }^{99} \mathrm{mTc}-\mathrm{MDP}$ in the} detection of breast cancer bone metastasis

\section{P Karan, AN Tiwari, R Pisal}

Nuclear Medicine, Department of Radiation Oncology, Kamla Nehru Hospital, Gandhi Medical College, Bhopal, India

Breast Cancer Research 2007, 9(Suppl 1):P24 (doi: 10.1186/bcr1730)

Finding bone metastases is frequently important for clinical decisions affecting quality of life. Detection of such bone metastatic lesions allows radiation therapy or surgical interventions to prevent pathologic fractures from disabling the patients. We investigated 53 breast cancer patients referred by surgeons, radiotherapists and chemotherapists. Adsorption of ${ }^{99} \mathrm{mTc}-\mathrm{MDP}$ occurs in the mineral phase of the bone. High-sensitivity bone scanning in determining the presence and extent of metastatic disease is an extremely important tool in decision-making. Bone scans demonstrate metastatic lesions much earlier than X-ray, CT or MRI. ${ }^{99} \mathrm{mTc}-\mathrm{MDP} 22-25 \mathrm{mCi}$ was injected i.v. In total, 53 patients were investigated. On visual analysis there were positive scan findings (bone mets) in 17 patients (33.4\%) and negative scan findings (normal bone scan) in 34 patients $(66.6 \%)$. Significance of bone mets in the management of disease occurs when clinically stage $I$ and scintigraphically stage IV; here a change of treatment is needed. Sensitivity for detecting bone mets is quoted as $95 \%$ or above. Bone scintigraphy is a noninvasive technique used for detection of breast cancer bone metastasis. Quality of life will be good if diagnosed early and accurately and if managed properly. 